\title{
Co-optimization of Distributed Renewable Energy and Storage Investment Decisions in a TSO-DSO Coordination Framework
}

This paper was downloaded from TechRxiv (https://www.techrxiv.org).

\section{LICENSE}

CC BY-NC-SA 4.0

SUBMISSION DATE / POSTED DATE

03-03-2022 / 08-03-2022

\section{CITATION}

Steriotis, Konstantinos; Makris, Prodromos; Tsaousoglou, Georgios; Efthymiopoulos, Nikos; Varvarigos, Emmanouel (2022): Co-optimization of Distributed Renewable Energy and Storage Investment Decisions in a TSO-DSO Coordination Framework. TechRxiv. Preprint. https://doi.org/10.36227/techrxiv.19298513.v1

$\mathrm{DOI}$

10.36227/techrxiv.19298513.v1 


\title{
Co-optimization of Distributed Renewable Energy and Storage Investment Decisions in a TSO-DSO Coordination Framework
}

\author{
K. Steriotis, P. Makris, G. Tsaousoglou, N. Efthymiopoulos, and E. Varvarigos
}

\begin{abstract}
As Distributed Energy Resources (DER) penetration levels and distributed flexibility investments are continuously growing, various smart grid actors need to coordinate their decisions towards optimal DER siting and sizing: First, profitbased Energy Service Providers (ESPs) want to secure their long-term profits and avoid economically unsustainable DER investments. Second, Distribution System Operators (DSOs) need to ensure the reliable operation of their networks in an economically optimal way. Third, Transmission System Operators (TSOs) want to optimally exploit the available "clean" DERs in close collaboration with the downstream DSOs. In this paper, we propose a novel ESP-DSO-TSO coordination scheme to cooptimize distributed renewable energy and storage planning at the distribution network level, while modeling the coordinated TSO-DSO operations. We formulate a bi-level program, the upper-level of which represents the minimization of DSO's costs, ensuring a minimum rate of return on ESP's investments, while the lower-level problem models the wholesale market clearing at the transmission network level. A nested decomposition technique is used to achieve computational tractability. Simulation results showcase a trade-off analysis between sustainable DER investments and system cost minimization and prove that an ESP-DSO-TSO interaction can benefit all involved actors to a certain extent. Finally, the computational efficiency of the proposed nested decomposition algorithm is also demonstrated via numerical results.
\end{abstract}

Index Terms-distributed energy resources, sizing and siting, bi-level optimization, nested decomposition, TSO-DSO coordination

\section{NOMENCLATURE}

A. Sets

$B_{i}$

$H$

$L_{i}^{D}$

$L^{T}$

$N$

$X^{U / L / M P}$

$\Omega$

\section{B. Superscripts}

$e / p$

Superscript indicating the energy/power component of ES

This work has been submitted to the IEEE for possible publication. Copyright may be transferred without notice, after which this version may no longer be accessible.

This work received funding from the European Union's Horizon 2020 research and innovation programme under grant agreement No. 863876 in the context of the FLEXGRID project. $e s / w / p v$

$m / g / d$

$(\cdot)$

C. Variables

dis/ch

$f^{p / q}$

$g^{w / p v}$

$h$

$K$

$o / b$

$p^{\uparrow / \downarrow}$

$p^{g / d}$

SOE

V

$x$

$z / \xi$

$\gamma_{\omega} / \mu$

$\theta$

$\lambda$

$\phi$

D. Parameters

$c^{\uparrow / \downarrow}$

$c^{e s / w / p v}$

$c^{g / d}$

C

$\tilde{C}$

$C^{i n v, \max }$

D

$f^{S}$

$I / W$

$\bar{K}$
Superscripts indicating the Storage/Wind/PV technology

Superscripts indicating TN nodes with DNs/Generators/Demand Aggregators

Superscript indicating the algorithm's iteration

Scheduled ES discharge/charge power (MW)

Active/reactive power flow in distribution network (MW/MVAr)

Power output of wind/PV units (MW)

Binary variable denoting the direction of power traded between DSO-TSO

Size variables

Quantity offer/bid by the DSO to the electricity market (MW)

Power traded (sold/bought) between DSO-TSO (MW)

Electricity market dispatch of Generators/Demand Aggregators (MW)

State-of-Energy of ES (MWh)

Square voltage magnitude in distribution network $\left(k V^{2}\right)$

Binary variable denoting the operating mode of ES

Auxiliary variables in outer decomposition Auxiliary variables in inner decomposition Transmission network's voltage phase angle Locational Marginal Price (€/ MW)

Dual variables of the lower-level problem

Price offers/bids of DSO (€/MW)

Marginal operating cost of ES/Wind/PV units $(€ / \mathrm{MW})$

Price bids of Generators/Demand Aggregators (€/MW)

Capital costs (€/MW)

Annualized capital costs (€/MW)

Investment budget ( $€$ )

Load of distribution network (MW)

Maximum apparent power of distribution network branches (MVA)

PV energy output/Wind intensity factor Maximum capacity that can be installed (MW) 


\begin{tabular}{|c|c|}
\hline$\overline{p_{i}}$ & $\begin{array}{l}\text { TSO-DSO connection point (substation) capac- } \\
\text { ity (MW) }\end{array}$ \\
\hline$\overline{P^{g / d}}$ & Maximum power of generation/demand (MW) \\
\hline$r / x$ & Resistance / Reactance of branches \\
\hline$R U / R D$ & $\begin{array}{l}\text { Ramping up/down capabilities of Generators } \\
\text { (MW) }\end{array}$ \\
\hline$T$ & Last timeslot of scheduling horizon \\
\hline $\bar{T}$ & Transmission network line capacity (MW) \\
\hline$\underline{V} / \bar{V}$ & $\begin{array}{l}\text { Lower/Upper bounds on square voltage mag- } \\
\text { nitude }\left(k V^{2}\right)\end{array}$ \\
\hline$y$ & Transmission line admittance \\
\hline$\beta$ & $\begin{array}{l}\text { Minimum ES state of charge at the end of the } \\
\text { scheduling period }\end{array}$ \\
\hline$\gamma^{\min }$ & Large negative constant \\
\hline$\delta$ & $\begin{array}{l}\text { Parameters converting active power into their } \\
\text { reactive power }-\tan (\arccos \text { (power factor) })\end{array}$ \\
\hline$\epsilon_{1} / \epsilon_{2}$ & $\begin{array}{l}\text { Convergence tolerance for the inner/outer de- } \\
\text { composition procedures }\end{array}$ \\
\hline$\eta^{d / c}$ & Discharging/Charging efficiency of ES \\
\hline$\eta^{p v}$ & PV efficiency \\
\hline$\kappa, \rho, v, \tau$ & Algorithm iteration counters \\
\hline$\pi_{\omega}$ & Weight of representative day $\omega$ \\
\hline$\rho$ & Energy/Power ratio of ES \\
\hline$\Phi$ & Large constant \\
\hline$\chi$ & Desired rate of return on DERs investment \\
\hline
\end{tabular}

\section{INTRODUCTION}

\section{A. Motivation and Goals}

Regulatory authorities from the world's most developed economies have undertaken clean energy transition initiatives and respective legislative efforts, towards actively incentivizing investments in Distributed Energy Resources (DERs) e.g. photovoltaics, wind turbines, etc., and distributed flexibility (e.g. battery, storage, etc.) installations at the distribution network (DN) level [1] - [3]. For example, US FERC [1] emphasizes the need for coordinated DER planning via the collaboration of three main actors, namely: the ISO/RTO (i.e. transmission level), the distribution utility, and the DER aggregator. In EU legislation [2], the DSO's main role is unbundled from profit-based Energy Service Providers (ESPs), i.e. DER and distributed flexibility owners. In other words, ESPs are the main responsible market actors for DER and distributed flexibility investments, while DSOs are mainly responsible for operating the $\mathrm{DN}$ in a reliable, secure and economically efficient manner. As a result, ESPs and DSOs should closely collaborate towards DN-aware DER investments, while DSOs should closely collaborate with TSOs in order to facilitate seamless DER market participation. Complementarily, [3] also focuses on investment-friendly and system-friendly renewable energy deployments implying the need for achieving a balance between these two contradictory objectives.

In this paper, we deal with the co-optimization of distributed renewable energy and storage investment problem (i.e. optimal sizing and siting of distributed PV, wind and storage assets), while modeling also the operational stage of distribution and transmission network levels. We formulate a bi-level model, in which the payoff of all DN users is optimized, to incentivize
DERs profitability and maximum exploitation of distributed energy and flexibility services in both DN and TN levels. The central contribution lies in the modeling of an integral ESPDSO-TSO coordination scheme, which jointly addresses the current real-life business challenges of these three actors. More specifically, the main problem of actual ESPs is not having access to detailed DN topology data, which leads them to inadequate, sub-optimal and/or financially unsustainable DER and flexibility investments. On the other hand, the DSOs cannot provide access to sensitive network topology data to profitbased ESPs and would strongly prefer to have full control of the DN-level investment planning process. However, regulated investments would dis-incentivize merchant DER investments and would be in contradiction with previously mentioned regulatory directives that press the DSOs towards providing the appropriate transparent, non-discriminatory and marketbased procedures for procuring novel energy and flexibility services from profit-based ESPs [2]. Finally, today's TSO problem is that it cannot optimally exploit the available DERs and DN-level flexibility due to lack of an efficient TSODSO coordination framework and because profit-based DER investments are $\mathrm{DN}$-unaware.

Three main research threads can be identified in the related literature. The first thread deals with ESP-TSO coordination schemes for optimal RES and/or storage sizing and siting at the transmission network (TN) level. The work in [4] proposes a Bender's decomposition algorithm to solve a bi-level problem, in which the upper-level problem represents the wind investment decisions and operations of the ESP, and the lower-level problems describe the market clearing under different wind and load conditions. Authors in [5] - [11] propose various bi-level models for optimal storage planning at the TN level together with novel decomposition techniques to deal with the computational complexity. Works [7] - [10] guarantee the ESP's profits ensuring a desirable Return-on-Investment (RoI) considering the storage asset's market participation with respect to the asset's expected lifetime. In [8] and [11], the risk management problem of the $\mathrm{TN}$-level storage investment is also investigated, trying to achieve an acceptable trade-off between maximum (average) expected profits and minimum guaranteed profits for the ESP's investment portfolio. Authors in [12] co-optimize both RES and storage planning via participation in day-ahead, intra-day energy and reserve markets. While the studies mentioned in this paragraph model assets connected to the $\mathrm{TN}$, in this paper we consider investments in DN-level assets. Under this consideration, it is critical to model the DN topology constraints [13] in order to guarantee DN reliability and security and ultimately avoid miscalculations of the DN-level energy and flexibility value [14].

The second related research thread deals with TSO-DSO coordination schemes. Survey works in [15] and [16] categorize several TSO-DSO coordination schemes and analyze the advantages and drawbacks for each one of them with respect to business interest prioritization of each actor. More technical papers such as [17] and [18] try to realistically model the information flows between the TSO and DSO by proposing decomposition algorithms for the coordinated economic dispatch of both $\mathrm{TN}-$ and DN-level systems that 
can capture the heterogeneous technical characteristics of both systems. Authors in [19] were the first to propose a modelling framework for the coordinated storage investment under such a unified TSO-DSO collaboration scheme. However, [19] does not consider a co-optimized planning of distributed renewable energy and storage assets, nor does it consider the latest regulations (e.g. [2]) dictating the unbundling of profit-based ESPs and non-profit DSOs. In contrast, this work emphasizes the need to quantify the impact of privately-owned DER investment decisions on the Transmission and Distribution (T\&D) systems' operating costs by aspiring to find an optimal equilibrium point that satisfies all three involved actors.

Finally, the third research thread is related with ESP-DSO coordination schemes. The main difference with the current manuscript's approach is that related works [20] - [25] do not take into account the TSO's objective to optimally exploit the available distributed energy and flexibility. Thus, a rather myopic DER planning is realized, that does not consider the impact of the newly installed low-cost energy and flexibility units on the transmission-level market prices. The work in [20] deals with a two-stage stochastic problem, in which initially the DSO minimizes its DN reinforcement costs and in the second stage the ESP's planning and scheduling problem takes place. Study [21] co-optimizes the ESP's and DSO's investment decisions showing that the ESP's flexibility significantly defers costly network upgrades. Authors in [22] achieve an optimal trade-off between the mobile storage profits during normal system operations and their ability to enhance DN resilience in emergency situations. Research work in [23] formulates an optimization problem in a way that includes both the ESP's profits and the DN constraints that guarantee power quality. Finally, [24] and [25] propose bi-level models to achieve the collaborative optimization between planning and operation of DN-level storage assets.

\section{B. Paper Positioning and Contribution}

This paper's contributions can be summarized as follows:

1) We co-optimize network-aware and market-aware distributed RES and storage investments in multiple DNs within a TSO-DSO coordination scheme.

2) Instead of modeling a vertical utility actor's (e.g. DISCO) objective in the upper-level problem, we follow the recent regulatory framework updates, which dictate the complete unbundling of market roles for DER investment planning between a profit-based ESP and a system operator. Thus, we conduct a trade-off analysis between sustainable DER investments and system cost minimization and demonstrate that ESP-DSO-TSO interaction can achieve T\&D operating costs' reduction and sufficient levels of profitability for the ESP.

3) We formulate a stochastic bi-level investment model in order to capture the impact of the newly installed DERs on the transmission-level energy market prices and avoid over-investment contexts.

4) The bi-level model is efficiently solved in a scalable fashion using a nested decomposition technique. We compare the computational performance of the proposed method to a non-decomposed MILP approach and demonstrate the algorithm's accuracy and scalability.

This paper's structure is organized as follows: Section III describes the proposed bi-level formulation of the problem. Section III presents the solution method. Section IV provides a detailed evaluation of the proposed solution. Finally, Section $\mathrm{V}$ concludes the paper and discusses future work.

\section{SyStem MOdEL AND PROBlem Formulation}

We model the interaction between a DSO, a TSO and a private ESP that seeks to invest in distributed wind and PV generation units as well as in Energy Storage (ES) units in multiple DNs dispersed in various geographical areas (TN nodes) and make profit by participating in the TN-level electricity market. The DSO decides on the DER investments with respect to its network limitations and ensuring that its decisions are financially sustainable for the ESP. To this end, we propose a stochastic bi-level model in order to formulate the DSO-TSO-ESP coordination, capturing their distinctive objectives and taking into account the DN and TN constraints.

The objective of the upper level problem, is to decide the new investments, so as to minimize the total costs (maximize social welfare) of the DNs' stakeholders, i.e., achieving a balance between the ESP's profits from the new investments and the electricity cost of consumers already connected to each DN. The former consists of the ESP's expected revenues stemming from the optimal DA market participation minus the operational costs and investment costs. Note that the relevant optimal operational decisions are contingent on the investment decisions as well as the DN constraints, including power flow and voltage limits. The objective of the ESP is to guarantee the economic viability of its investments. Therefore, a minimum rate of return on the investments is imposed on the upper-level problem.

The lower-level problem represents the TN-constrained dayahead electricity market clearing process for each representative day, with the TSO's objective being the social welfare maximization. Thus, the objective of all involved actors (DSOTSO-ESP) are taken into account. In order to model the uncertainty associated with the overall system's load, the DN's demand and the wind/solar production, we produce a plausible number of scenarios by using data of diverse representative historical days. As it is customary in static investment analysis (e.g. [4]- [7], [9], [10]), the study is performed for a single target year.

\section{A. Upper-Level Problem: Siting and Sizing of DERs}

$$
\min _{X_{S}^{U} \cup X_{O, \omega}^{U} \cup X_{\omega}^{L}, \forall \omega} \sum_{\omega \in \Omega}\left(-P r_{\omega}^{D N}+C_{\omega}^{o p e r}\right)+\tilde{C}^{i n v}
$$

where

$$
\begin{aligned}
& \operatorname{Pr}_{\omega}^{D N}=\pi_{\omega} \cdot \sum_{i \in N^{m}} \sum_{t \in H}\left(\lambda_{i t \omega} \cdot\left(p_{i t \omega}^{\uparrow}-p_{i t \omega}^{\downarrow}\right)\right) \\
& C_{\omega}^{\text {oper }}=\pi_{\omega} \cdot \sum_{i \in N^{m}}\left(\sum _ { t \in H } \left(\sum_{n \in B_{i}^{e s}} c^{e s} \cdot\left(d i s_{i n t \omega}+c h_{i n t \omega}\right)+\right.\right.
\end{aligned}
$$




$$
\begin{aligned}
& \left.\left.\sum_{n \in B_{i}^{w}} c^{w} \cdot g_{i n t \omega}^{w}+\sum_{n \in B_{i}^{p v}} c^{p v} \cdot g_{i n t \omega}^{p v}\right)\right) \\
& \tilde{C}^{i n v}=\sum_{i \in N^{m}}\left(\sum_{n \in B_{i}^{e s}}\left(\tilde{C}^{e} \cdot K_{i n}^{e}+\tilde{C}^{p} \cdot K_{i n}^{p}\right)+\sum_{n \in B_{i}^{w}} \tilde{C}^{w} \cdot K_{i n}^{w}\right. \\
& \left.+\sum_{n \in B_{i}^{p v}} \tilde{C}^{p v} \cdot K_{i n}^{p v}\right)
\end{aligned}
$$

\section{Subject to}

$$
\begin{aligned}
& 0 \leq g_{i n t \omega}^{w} \leq W_{i t \omega} \cdot K_{i n}^{w}, \quad \forall i \in N^{m}, n \in B_{i}^{w}, t \in H, \omega \in \Omega \\
& 0 \leq g_{i n t \omega}^{p v} \leq \eta^{p v} \cdot I_{i t \omega} \cdot K_{i n}^{p v}, \\
& \forall i \in N^{m}, n \in B_{i}^{p v}, t \in H, \omega \in \Omega \\
& 0 \leq K_{i n}^{w} \leq \overline{K^{w}}, \quad \forall i \in N^{m}, n \in B_{i}^{w} \\
& 0 \leq K_{i n}^{p v} \leq \overline{K^{p v}}, \quad \forall i \in N^{m}, n \in B_{i}^{p v} \\
& 0 \leq K_{i n}^{e} \leq \overline{K^{e}}, \quad \forall i \in N^{m}, n \in B_{i}^{e s} \\
& 0 \leq K_{i n}^{p} \leq \overline{K^{p}}, \quad \forall i \in N^{m}, n \in B_{i}^{e s} \\
& \rho \cdot K_{i n}^{p}=K_{i n}^{e}, \quad \forall i \in N^{m}, n \in B_{i}^{e s} \\
& C^{i n v} \leq C^{i n v, m a x} \\
& \text { where } \\
& C^{i n v}=\sum_{i \in N^{m}}\left(\sum_{n \in B^{e s}}\left(C^{e} \cdot K_{i n}^{e}+C^{p} \cdot K_{i n}^{p}\right)+\sum_{n \in B_{i}^{w}} C^{w} \cdot K_{i n}^{w}\right. \\
& \left.+\sum_{n \in B^{p v}} C^{p v} \cdot K_{i n}^{p v}\right) \\
& \sum_{\omega \in \Omega}\left(\operatorname{Pr}_{\omega}^{i n v}-C_{\omega}^{\text {oper }}\right) \geq \chi \cdot \tilde{C}^{i n v}
\end{aligned}
$$

where

$$
\begin{aligned}
& \operatorname{Pr}_{\omega}^{i n v}=\pi_{\omega} \cdot \sum_{i \in N^{m}} \sum_{t \in H}\left(\lambda _ { i t \omega } \cdot \left(\sum_{n \in B_{i}^{w}} g_{i n t \omega}^{w}+\sum_{n \in B_{i}^{p v}} g_{i n t \omega}^{p v}\right.\right. \\
& \left.\left.+\sum_{n \in B_{i}^{e s}}\left(d i s_{i n t \omega}-c h_{i n t \omega}\right)\right)\right) \\
& 0 \leq o_{i t \omega} \leq h_{i t \omega} \cdot \overline{p_{i}}, \\
& 0 \leq b_{i t \omega} \leq\left(1-h_{i t \omega}\right) \cdot \overline{p_{i}}, \quad \forall i \in N^{m}, t \in H, \omega \in \Omega \\
& h_{i t \omega} \in\{0,1\}, \quad \forall i \in N^{m}, t \in H, \omega \in \Omega
\end{aligned}
$$

$0 \leq d i s_{\text {int } \omega}, c h_{\text {int } \omega} \leq K_{i n}^{p}, \forall i \in N^{m}, n \in B_{i}^{e s}, t \in H, \omega \in \Omega$

dis $_{\text {int } \omega} \leq \Phi \cdot x_{i n t \omega}, \quad \forall i \in N^{m}, n \in B_{i}^{e s}, t \in H, \omega \in \Omega$

ch $_{\text {int } \omega} \leq \Phi \cdot\left(1-x_{\text {int } \omega}\right), \quad \forall i \in N^{m}, n \in B_{i}^{e s}, t \in H, \omega \in \Omega$

$x_{i n t \omega} \in\{0.1\}, \quad \forall i \in N^{m}, n \in B_{i}^{e s}, t \in H, \omega \in \Omega$

$$
S O E_{i n t \omega}=S O E_{i n 0 \omega}-\sum_{\tau=1}^{t}\left(d i s_{i n t \omega} / \eta^{d}-c h_{i n t \omega} \cdot \eta^{c}\right),
$$

$\forall i \in N^{m}, n \in B_{i}^{e s}, t \in H, \omega \in \Omega$

$0 \leq S O E_{\text {int } \omega} \leq K_{\text {in }}^{e}, \quad \forall i \in N^{m}, n \in B_{i}^{e s}, t \in H, \omega \in \Omega$

$S O E_{i n T \omega} \geq \beta \cdot S O E_{i n 0 \omega}, \quad \forall i \in N^{m}, n \in B_{i}^{e s}, \omega \in \Omega$

$$
\sum_{k \in \Omega_{d}(n)} f_{i(n k) t \omega}^{p}=\sum_{j \in \Omega_{p}(n)} f_{i(j n) t \omega}^{p}-D_{i n t \omega}+g_{i n t \omega}^{w}+g_{i n t \omega}^{p v}
$$$$
+d i s_{i n t \omega}-c_{i n t \omega}, \quad \forall i \in N^{m}, n \in B_{i}, t \in H, \omega \in \Omega
$$

$\sum_{k \in \Omega_{d}(n)} f_{i(n k) t \omega}^{q}=\sum_{j \in \Omega_{p}(n)} f_{i(j n) t \omega}^{q}-\delta_{i n}^{d} \cdot D_{i n t \omega}+\delta_{i n}^{w} \cdot g_{i n t \omega}^{w}$

$+\delta_{i n}^{p v} \cdot g_{i n t \omega}^{p v}, \quad \forall i \in N^{m}, n \in B_{i}, t \in H, \omega \in \Omega$

$V_{i n t \omega}=V_{i j t \omega}-2 \cdot\left(r_{i(j n)} \cdot f_{i(j n) t \omega}^{p}+x_{i(j n)} \cdot f_{i(j n) t \omega}^{q}\right)$,

$\forall i \in N^{m}, n \in B_{i}, t \in H, \omega \in \Omega$

$\left(f_{i(n k) t \omega}^{p}\right)^{2}+\left(f_{i(n k) t \omega}^{q}\right)^{2} \leq\left(f_{i(n k)}^{S}\right)^{2}$,

$\forall i \in N^{m},(n k) \in L_{i}^{D}, t \in H, \omega \in \Omega$

$\underline{V_{\text {in }}} \leq V_{\text {int } \omega} \leq \overline{V_{\text {in }}}, \quad \forall i \in N^{m}, n \in B_{i}, t \in H, \omega \in \Omega$

$\sum_{k \in \Omega_{d}\left(n_{0}\right)} f_{i\left(n_{0} k\right) t \omega}^{p}=p_{i t \omega}^{\downarrow}-p_{i t \omega}^{\uparrow}, \quad \forall i \in N^{m}, t \in H, \omega \in \Omega$

where $X_{S}^{U}=\left\{K_{i n}^{e}, K_{i n}^{p}, K_{i n}^{w}, K_{i n}^{p v}\right\}, X_{O, \omega}^{U}=\left\{g_{i n t \omega}^{w}, g_{i n t \omega}^{p v}\right.$, $o_{i t \omega}, b_{i t \omega}, h_{i t \omega}, d i s_{i n t \omega}, c h_{i n t \omega}, x_{i n t \omega}, S O E_{i n t \omega}, f_{i(n k) t \omega}^{p}$,

$\left.f_{i(n k) t \omega}^{q}, V_{i n t \omega}\right\}$. The objective of the upper-level problem a.1) is to minimize the annualized DER investment costs and the DN assets' expected costs, the latter including the expected cost of electricity traded with the upstream grid and the expected DER operating costs. The set of DNs in which DERs can be installed is denoted by $N^{m}$. The overall DN assets' expected profit from the electricity traded in the day-ahead electricity market are defined in Eq. a.2, where the nodal prices at the transmission buses connected to the root nodes of the DNs $\left(\lambda_{i t \omega}\right)$ and the wholesale market decisions on the power injected from the DSO to the TSO $\left(p_{i t \omega}^{\uparrow}\right)$ and vice versa $\left(p_{i t \omega}^{\downarrow}\right)$ are endogenously obtained from the lower-level problem. The expected DERs' operating costs are presented in a.3 and the annualized investment cost is computed in a.4, where parameters $\tilde{C}^{e}, \tilde{C}^{p}, \tilde{C}^{w}$ and $\tilde{C}^{p v}$ are the annualized costs in net present value approach [14]:

$$
\tilde{C}=C \cdot \frac{\Gamma \cdot(1+\Gamma)^{\Lambda}}{(1+\Gamma)^{\Lambda}-1}
$$

where $\Gamma$ is the annual discount rate and $\Lambda$ is the unit's lifetime. The sets of DN's buses that are eligible for the installation of wind/PV generation and ES units are $B_{i}^{w}, B_{i}^{p v}$ and $B_{i}^{e s}$ respectively $\left(B_{i}^{w}, B_{i}^{p v}, B_{i}^{e s} \subseteq B_{i}, \forall i \in N^{m}\right)$. Constraints a.5 and a.6) limit the wind and solar generation to the wind and solar power availability. Wind power availability is calculated based on a wind intensity factor [4], while the PV maximum output in a representative day is calculated based on PV energy output factor and the efficiency of the PV panels output [26]. Note that in our model, renewable energy spillage is allowed.

Constraints a.7 - a.10 limit the available capacity of wind, PV, ES energy and ES power capacity respectively, while constraint a.11 enforces the Energy-to-Power ratio of the ES units. For the sake of simplicity, the investment variables are considered continuous. Equation a.12 enforces that the total investment cost, which is shown in (a.13), cannot exceed the total ESP's investment budget. Constraint (a.14) enforces that the ESP's expected annual financial benefit gained 
from the investment, which is expressed in a.15), is sufficient to provide a certain desired rate of return $\chi$. Constraints a.16 - a.18 limit the offer/bid quantity $\left(o_{i t \omega} / b_{i t \omega}\right)$ in the wholesale market by the DN assets with respect to the capacity of the substations connecting the transmission and distribution networks. Binary variable $h_{i t \omega}(\mathrm{a} .18)$ ensures that the DNs can either supply or draw power from the main grid for a specific timeslot in each representative day. Constraints a.19-a.22 impose the bounds on the charge/discharge schedules of the ES units. Equation a.23 describes the State-of-Energy of the ES units, which is limited in a.24. Also, constraint a.25 declares that the ES units' State-of-Energy at the end of the scheduling horizon must be at least equal to a portion $(\beta)$ of their initial State-of-Energy at the beginning of the day.

The DN model is described in Eqs. a.26 - a.31. We use the linearized DistFlow equations [27] to model the DN. Equations a.26 - a.28 are the branch flow equations. Constraint a.29 sets the apparent power capacity of the lines and constraint a.30 sets the bounds of the square voltage magnitude. Constraint a.29 is a quadratic inequality constraint, which is linearized via a polygonal inner approximation as in [28]. Finally, equation a.31 represents the active power balance in the root of the DN, i.e., the connection point to the transmission grid.

\section{B. Lower-Level Problem: Day-Ahead Electricity Market} Clearing Process

$$
\begin{gathered}
\left\{\operatorname { m i n } _ { X _ { \omega } ^ { L } } \sum _ { t \in H } \left(\sum_{i \in N^{g}} c_{i t}^{g} \cdot p_{i t \omega}^{g}-\sum_{i \in N^{d}} c_{i t}^{d} \cdot p_{i t \omega}^{d}+\right.\right. \\
\left.\quad+\sum_{i \in N^{m}}\left(c_{i t}^{\uparrow} \cdot p_{i t \omega}^{\uparrow}-c_{i t}^{\downarrow} \cdot p_{i t \omega}^{\downarrow}\right)\right)
\end{gathered}
$$

Subject to

$$
\begin{aligned}
& -p_{i t \omega}^{g}+p_{i t \omega}^{d}-p_{i t \omega}^{\uparrow}+p_{i t \omega}^{\downarrow}+\sum_{j \neq i} y_{i j} \cdot\left(\theta_{i t \omega}-\theta_{j t \omega}\right)=0 ; \\
& \left(\lambda_{i t \omega}\right), \quad \forall i \in N, t \in H \\
& 0 \leq p_{i t \omega}^{g} \leq \overline{P_{i}^{g}} ; \quad\left(\underline{\phi_{i t \omega}^{g}}, \overline{\phi_{i t \omega}^{g}}\right), \quad \forall i \in N^{g}, t \in H \\
& R D_{i} \leq p_{i t \omega}^{g}-p_{n(t-1) \omega}^{g} \leq R U_{i} ; \quad\left(\phi_{i t \omega}^{g r d}, \phi_{i t \omega}^{g r u}\right), \\
& \forall i \in N^{g}, t>1 \\
& R D_{i} \leq p_{i t \omega}^{g}-p_{n, 0}^{g} \leq R U_{i} ; \quad\left(\phi_{i t \omega}^{g r d}, \phi_{i t \omega}^{g r u}\right), \\
& \forall i \in N^{g}, t=1 \\
& 0 \leq p_{i t \omega}^{d} \leq \overline{P_{i t \omega}^{d}} ; \quad\left(\underline{\phi_{i t \omega}^{d}}, \overline{\phi_{i t \omega}^{d}}\right), \quad \forall i \in N^{d}, t \in H \\
& 0 \leq p_{i t \omega}^{\uparrow} \leq o_{i t \omega} ; \quad\left(\underline{\phi_{i t \omega}^{p \uparrow}}, \overline{\phi_{i t \omega}^{p \uparrow}}\right), \quad \forall i \in N^{m}, t \in H \\
& 0 \leq p_{i t \omega}^{\downarrow} \leq b_{i t \omega} ; \quad\left(\underline{\phi_{i t \omega}^{p \downarrow}}, \overline{\phi_{i t \omega}^{p \downarrow}}\right), \quad \forall i \in N^{m}, t \in H \\
& -\overline{T_{i j}} \leq y_{i j} \cdot\left(\theta_{i t \omega}-\theta_{j t \omega}\right) \leq \overline{T_{i j}} ; \quad \underline{\left(\phi_{(i j) t \omega}^{l}, \overline{\phi_{(i j) t \omega}^{l}}\right)}, \\
& \forall(i j) \in L^{T}, i<j, t \in H \\
& \}, \forall \omega \in \Omega
\end{aligned}
$$

where $X_{\omega}^{L}=\left\{p_{i t \omega}^{g}, p_{i t \omega}^{d}, p_{i t \omega}^{\uparrow}, p_{i t \omega}^{\downarrow}, \theta_{i t \omega}\right\}$. In the lower-level problem (b), the TSO clears the wholesale day-ahead electricity market for each representative day $\omega$, by minimizing the social cost (or else maximizing the social welfare), given the DN assets' supply offers $\left(c_{i t}^{\uparrow}\right)$ and demand bids $\left(c_{i t}^{\downarrow}\right)$, offers from generators $\left(c_{i t}^{g}\right)$ and bids from demand aggregators $\left(c_{i t}^{d}\right)$. The transmission network is modeled using the DC power flow model. Equation b.2 expresses the nodal power balance, while constraints (b.3) and b.6) set the active power capacities of conventional generators and demand aggregators respectively. Constraints (b.4) - b.5) express the ramping capabilities of the conventional generators and equations (b.7) - b.8 limit the power traded between the TN and the DNs. Finally, constraint (b.9) binds the active power flow in the transmission lines. The dual variables pertaining to each constraint are specified following a semicolon.

\section{Solution Method}

The formulated stochastic bi-level program can be solved through converting it into a Mathematical Program with Equilibrium Constraints (MPEC) and eventually into a MILP, as in [9], [19], [29] and [30]. We denote the optimal value of this single-level mixed integer linear problem as $\Psi_{P}$. The computational complexity of this method increases dramatically with the number of representative days. In order to circumvent intractability, we decompose the bi-level problem in representative days $\omega$. Note that the ESP's minimum profit constraint a.14 and the investment variables $\left(K_{i n}^{w}, K_{i n}^{p v}, K_{i n}^{e}\right.$, and $K_{i n}^{p}$ ) prevent the decoupling of the problem per representative day. Thus, we apply a nested decomposition algorithm, which renders the problem computationally tractable. The outer decomposition algorithm deals with the complicating constraint a.14, while the inner decomposition procedure deals with the complicating variables $\left(K_{i n}^{w}, K_{i n}^{p v}, K_{i n}^{e}\right.$, and $\left.K_{i n}^{p}\right)$.

\section{A. Outer Decomposition: Relaxing the ESP's minimum profit constraint}

Initially, we deal with the complicating constraint a.14 using the Lagrangian Relaxation (LR) technique [31]. We relax constraint a.14 by removing it from the set of constraints inserting a penalty for violations. Let $\xi$ be a non-negative scalar and consider the following problem:

$$
\begin{array}{r}
\Psi_{D}(\xi)=\min _{X_{S}^{U} \cup X_{O, \omega}^{U} \cup X_{\omega}^{L}, \forall \omega} \sum_{\omega \in \Omega}\left(-P r_{\omega}^{D N}+C_{\omega}^{\text {oper }}\right)+\tilde{C}^{\text {inv }} \\
+\xi \cdot\left(\sum_{\omega \in \Omega}\left(-P r_{\omega}^{\text {inv }}+C_{\omega}^{\text {oper }}\right)+\chi \cdot \tilde{C}^{i n v}\right)
\end{array}
$$

Subject to

$$
a .5-a .13, a .16-a .31, b .1-b .9
$$

The above problem (c) is a relaxed version of problem (a)-(b). We convert the above bi-level problem into a MILP using the MPEC method as explained in APPENDIX A. It can be easily seen that the relaxed problem (c) provides a lower bound for the initial (non-relaxed) problem, i.e. $\Psi_{D}(\xi) \leq \Psi_{P}$, since $\sum_{\omega \in \Omega}\left(-\operatorname{Pr}_{\omega}^{i n v}+C_{\omega}^{\text {oper }}\right)+\chi \cdot \tilde{C}^{i n v} \leq 0$ and $\xi \geq 0$. Function $\Psi_{D}(\xi)$ is the dual function of problem (a)-(b). Our goal is to find the optimal value of $\xi$ that will result 


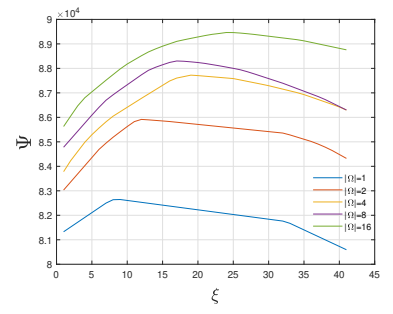

(a) Value of dual function $\Psi$ for different values of $\xi$ and various numbers of representative days $|\Omega|$.

Fig. 1: Simulations on a 6-Bus Transmission System and 15-node DN 33

in the best bound $\Psi_{D}\left(\xi^{*}\right)$ of $\Psi_{P}$, solving the optimization problem: $\max _{\xi}\left\{\Psi_{D}(\xi) \mid \xi \in \mathbb{R}^{+}\right\}$, which is the Lagrangian dual problem of the original problem.

Towards this objective, we use the LR decomposition algorithm. This is an iterative procedure, in each iteration of which the relaxed problem (c) is converted into a MILP (cf. APPENDIX A) and solved for a specific value of $\xi$. Given the optimal solution and the respective values of $\sigma=\left(\sum_{\omega \in \Omega}\left(-\operatorname{Pr}_{\omega}^{i n v}+C_{\omega}^{o p e r}\right)+\chi \cdot \tilde{C}^{i n v}\right)$, we update the value of $\xi$ using the Cutting Plane (CP) method [32]. This updating method reconstructs the dual function $\Psi_{D}(\xi)$, which is a concave piecewise linear function as it is demonstrated in Fig. 1a using cutting planes. More specifically, in outer-loop iteration $\kappa$, the value of $\xi$ is updated via solving the following linear program:

$$
\max _{z, \xi} z
$$

Subject to

$$
\begin{aligned}
& z \leq \Psi_{D}\left(\xi^{(\rho)}\right)+\sigma^{(\rho)} \cdot\left(\xi-\xi^{(\rho)}\right) ; \quad \rho=1, . ., \kappa \\
& \xi \geq 0
\end{aligned}
$$

Constraints d.2 represent hyperplanes in the variable $\xi$ space. Problem (d) is a relaxed version of the Lagrangian dual problem, which approaches the actual Lagrangian dual problem as the number of iterations increases. The optimal solution of problem (d) provides an upper bound of the optimal objective function value of the Lagrangian dual problem $\Psi_{D}\left(\xi^{*}\right)$, since the piecewise linear reconstruction of $\Psi_{D}(\xi)$ overestimates the actual function [32], i.e. $z^{(\kappa)} \geq \Psi_{D}\left(\xi^{*}\right) \geq$ $\Psi_{D}\left(\xi^{(\kappa)}\right)$. The right inequality follows from the fact that the optimal objective function value of the aforementioned Lagrangian dual maximization problem is always greater than or equal to any other feasible solution provided by the solution of problem (c). The algorithm terminates when the per unit gap is below a threshold, that is, $\frac{z^{(\kappa)}-\Psi_{D}\left(\xi^{(\kappa)}\right)}{\Psi_{D}\left(\xi^{(\kappa)}\right)} \leq \epsilon_{1}$.

Under convexity assumptions, the best bound $\Psi_{D}\left(\xi^{*}\right)$ is equal to the optimal value of $\Psi_{P}$ (Strong Duality Theorem). In general, however, it falls short. The difference between $\Psi_{D}\left(\xi^{*}\right)$ and $\Psi_{P}$ is the duality gap. In our model, the LR provides a high quality bound on $\Psi_{P}$. We confirm this claim by simulation on a test system in Fig. $1 \mathrm{~b}$ that the maximum duality gap (difference between $\Psi_{P}$ and $\Psi_{D}\left(\xi^{*}\right)$ ) is $0.11 \%$ (in case $|\Omega|=1$ ), while the average per unit gap is $0.03 \%$.

\section{B. Inner Decomposition: Bender's Decomposition Technique}

We now deal with the investment decision variables, namely $K_{i n}^{p v}, K_{i n}^{w}, K_{i n}^{e}$ and $K_{i n}^{p}$, that prevent the solution of the problem (c) by blocks (one for each representative day). We apply a Bender's Decomposition (BD) technique, that has been proposed in [34]. In general, the BD algorithm does not provide convergence guarantees for non-convex problems. Nevertheless, studies on the optimal investments in the power systems using stochastic bi-level modeling ([4], [5], [10], [34]) have shown that the objective function of the original (non-decomposed) problem convexifies with respect to the investment (complicating) variables as the number of scenarios and their diversity increases. The bi-level problem (c) is decomposed into a Master Problem (MP) and a number of subproblems, one per representative day. At first, the linear and continuous MP is solved and provides updated investment decisions. In BD algorithm's iteration $v$, the MP resulting from decomposing the original problem (c) follows:

$$
\min _{X^{M P}} \underline{G}^{(v)}=(1+\xi \cdot \chi) \cdot \tilde{C}^{i n v}+\sum_{\omega \in \Omega} \gamma_{\omega}^{(v)}
$$

Subject to

$a .7-a .13$

$\gamma_{\omega}^{(v)} \geq \gamma^{\min }, \quad \forall \omega \in \Omega$

$\gamma_{\omega}^{(v)} \geq \tilde{G}_{\omega}^{(v)}+\sum_{i \in N^{m}}\left(\sum_{n \in B_{i}^{w}} \mu_{i n \omega}^{w,(\tau)} \cdot\left(K_{i n}^{w,(v)}-K_{i n}^{w,(\tau)}\right)+\right.$

$\sum_{n \in B_{i}^{p v}} \mu_{i n \omega}^{p v,(\tau)} \cdot\left(K_{i n}^{p v,(v)}-K_{i n}^{p v,(\tau)}\right)+$

$\left.\sum_{n \in B_{i}^{e s}}\left(\mu_{i n \omega}^{e,(\tau)} \cdot\left(K_{i n}^{e,(v)}-K_{i n}^{e,(\tau)}\right)+\mu_{i n \omega}^{p,(\tau)} \cdot\left(K_{i n}^{p,(v)}-K_{i n}^{p,(\tau)}\right)\right)\right)$

$\forall \omega \in \Omega, \tau=1, .,, v-1$

where $X^{M P}=\left\{K_{i n}^{w,(v)}, K_{i n}^{p v,(v)}, K_{i n}^{e,(v)}, K_{i n}^{p,(v)}, \gamma_{\omega}^{(v)}\right\}$. In e.1 , $\gamma_{\omega}^{(v)}$ is a scalar representing the optimal value of the subproblem in representative day $\omega$, in BD algorithm's iteration $v$. Constraint e.3 imposes a lower bound on $\gamma_{\omega}^{(v)}$ to accelerate convergence. Constraints (e.4) are the Bender's cuts, where $\mu_{i n \omega}^{w}, \mu_{i n \omega}^{p v}, \mu_{i n \omega}^{e}$ and $\mu_{i n \omega}^{w}$ are sensitivities obtained from the subproblems' solution. Parameters including superscripts $(\tau)$ are obtained from the previous iterations. Note that at each iteration, we add multiple cuts to the MP (one for each $\omega$ ), in order to accelerate the decomposition procedure ([6], [35]). The solution of the MP updates the values of the DERs' sizes and location. The MP is a relaxed version of the original problem (c) and the objective function (e.1 approximates from below the objective function of the original problem $\left(\underline{G}^{v} \leq \Psi_{D}(\xi)\right)$. Then, for each representative day $\omega$, a subproblem is formulated:

$$
\min _{X_{O, \omega}^{U} \cup X_{\omega}^{L}} \tilde{G}_{\omega}=(1+\xi) \cdot C_{\omega}^{o p e r}-\operatorname{Pr}_{\omega}^{D N}-\xi \cdot \operatorname{Pr}_{\omega}^{i n v}
$$

Subject to

$$
\begin{aligned}
& \text { a.5 }, a .6, \quad a .16-a .31, b .1-b .9 \\
& K_{i n}^{w}=\tilde{K}_{i n}^{w} ; \quad\left(\mu_{i n \omega}^{w}\right), \quad \forall i \in N^{m}, n \in B_{i}^{w}
\end{aligned}
$$




$$
\begin{array}{llll}
K_{i n}^{p v}=\tilde{K}_{i n}^{p v} ; & \left(\mu_{i n \omega}^{p v}\right), & & \forall i \in N^{m}, n \in B_{i}^{p v} \\
K_{i n}^{e}=\tilde{K}_{i n}^{e} ; & \left(\mu_{i n \omega}^{e}\right), & & \forall i \in N^{m}, n \in B_{i}^{e s} \\
K_{i n}^{p}=\tilde{K}_{i n}^{p} ; & \left(\mu_{i n \omega}^{p}\right), & & \forall i \in N^{m}, n \in B_{i}^{e s}
\end{array}
$$

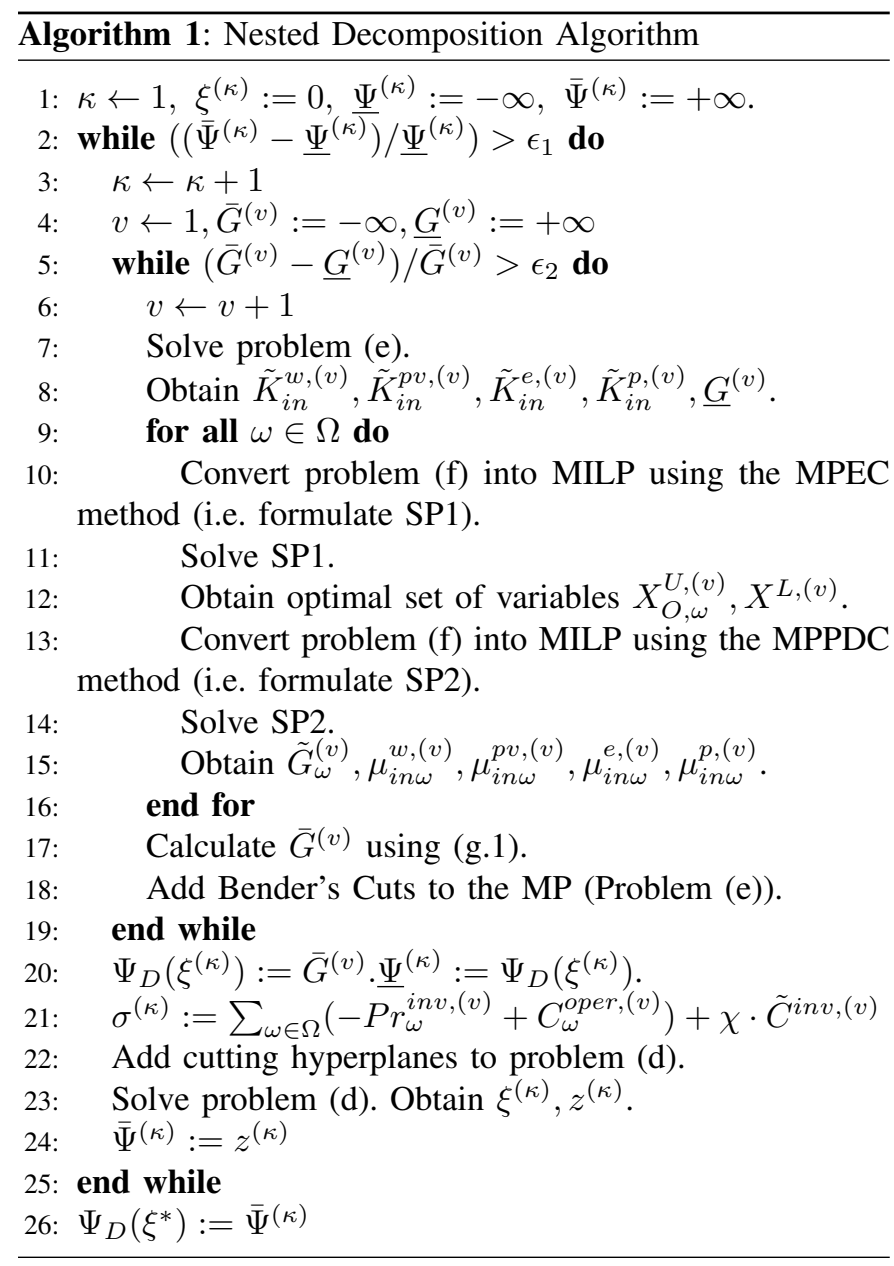

Note that in the above problem, all variables pertain to each algorithm's iteration $v$, but for notational clarity, such a superscript is omitted. The objective in the above problem $\left(\tilde{G}_{\omega}\right)$ is to minimize the operating costs of the DNs assets in representative day $\omega$ plus the part of the dualized ESP profit constraint that contains only daily operation variables. Equations (f.3) - f.6. fix the wind production, solar production, ES energy rating and ES power rating respectively, to the values computed in the MP. Consequently, the bi-level subproblems (f) are converted into MILPs with the MPEC method (SP1), as described in APPENDIX B. Since the solution of the MILPs does not provide accurate dual variables to build the Bender's cuts, we reformulate the subproblems into equivalent linear continuous problems, using the Mathematical Programming with Primal and Dual Constraints (MPPDC) formulation of the bi-level subproblems (SP2), as explained in APPENDIX C. Solving SP2 provides $\tilde{G}_{\omega}$ and dual variables $\mu_{i n \omega}^{w}, \mu_{i n \omega}^{p v}, \mu_{i n \omega}^{e}$ and $\mu_{i n \omega}^{p}$. The value of $\bar{G}^{(v)}$ to be used in the convergence check is:

$$
\bar{G}^{(v)}=\sum_{\omega \in \Omega} \tilde{G}_{\omega}^{(v)}+\sum_{i \in N^{m}}\left(\sum_{n \in B_{i}^{w}} \tilde{C}^{w} \cdot \tilde{K}_{i n}^{w,(v)}\right.
$$

$$
\left.+\sum_{n \in B_{i}^{p v}} \tilde{C}^{p v} \cdot \tilde{K}_{i n}^{p v,(v)}+\sum_{n \in B_{i}^{e s}}\left(\tilde{C}^{e} \cdot \tilde{K}_{i n}^{e,(v)}+\tilde{C}^{p} \cdot \tilde{K}_{i n}^{p,(v)}\right)\right)
$$

Each subproblem is a further restricted version of the original problem (c). Hence, its optimal objective function value is an upper bound to the optimal value of the objective function of the original problem $\left(\bar{G}^{(v)} \geq \Psi_{D}(\xi)\right)$. The procedure continues until $\left(\bar{G}^{(v)}-\underline{G}^{(v)}\right) / \bar{G}^{(v)} \leq \epsilon_{2}$. The details of the overall algorithm are described in Algorithm 1.

\section{CAse Study}

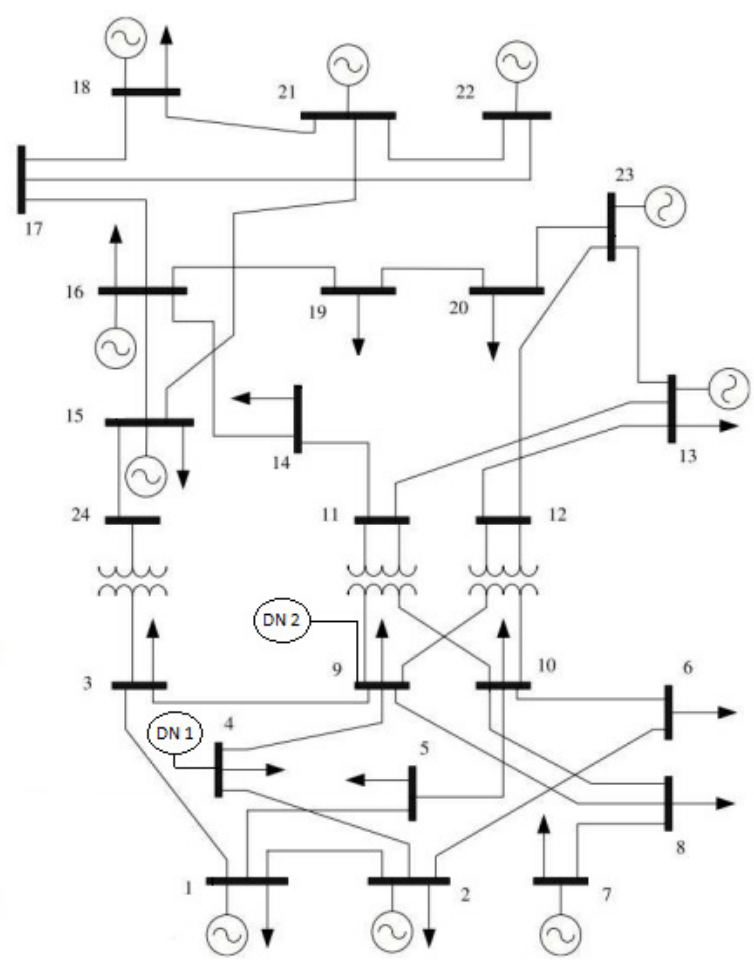

Fig. 2: IEEE One-Area Reliability Test System

The proposed methodology is tested on the IEEE One Area Reliability Test System [36], which is illustrated in Fig. 2 The generation, load and line data can be found in [37]. We consider that the DER investments can take place on two 33-bus distribution networks (see [38] for network details) with their root nodes being connected to transmission buses 4 and 9. Detailed distribution network data can be found in [37]. Load and renewable energy production profiles are based on realistic annual data from the European Network of Transmission System Operators for Electricity (ENTSOE) and the Hellenic Distribution Network Operator (HEDNO) respectively [37], which are reduced to eight representative days with 24 time intervals using the k-means clustering algorithm. The wind intensity and PV energy output factors were selected from two different locations (distribution networks) in Greece, while without loss of generality we assumed that the demand follows the same pattern in both the transmission and distribution network. The weighted average of demand, wind intensity and PV energy output scenarios are depicted in Fig. 3 The eligible distribution network nodes are presented 

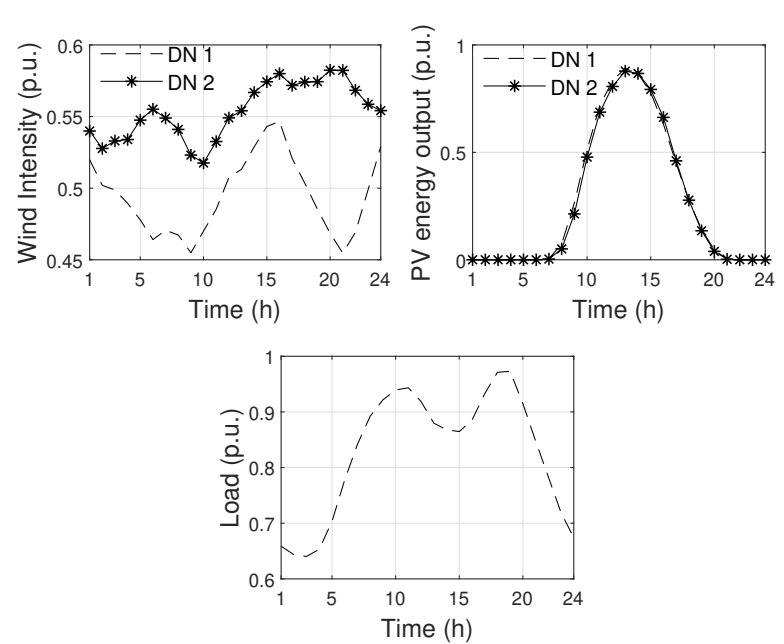

Fig. 3: Expected Values of Load, Wind Intensity and PV Energy Output

in Table I We assume that the capital costs of DERs are $C^{w}=1300 € / \mathrm{kW}, C^{p v}=830 € / \mathrm{kW}, C^{e}=20 € / \mathrm{kW}$ and $C^{p}=500 € / \mathrm{kW}$, with their marginal operating costs being $c^{w}=3.5 € / \mathrm{MW}, c^{p v}=2.5 € / \mathrm{MW}$ and $c^{e s}=0.5 € / \mathrm{MW}$. The lifetimes of DERs are 15 years and the annual discount rate is $5 \%(\Lambda=15, \Gamma=0.05)$. Due to several restrictions (e.g. limited available area), we set the maximum capacity of DERs to $\overline{K^{w}}=20 M W, \overline{K^{p v}}=10 M W$ and $\overline{K^{e}}=20 M W h$. The energy-to-power ratio of candidate storage units is set to $\rho=6 h$ and the charging and discharging efficiencies are $\eta^{c}=\eta^{d}=0.93$. The initial state-of-charge of the storage units is assumed to be $50 \%$, while at the end of the day the state-of-charge should be at least $10 \%(\beta=0.1)$. The power factor of the candidate wind and PV generating units is assumed to be 0.95 , while the efficiency of the PV panels output is $\eta^{p v}=0.95$. The maximum power flow on the coupling points between the transmission and distribution systems (substations) is set to $\overline{p_{i}}=46 \mathrm{MW}$. The price bids and offers of the DNs assets are set to $c_{i t}^{\downarrow}=450 € / \mathrm{MW}$ and $c_{i t}^{\uparrow}=0 € / \mathrm{MW}$ in order to ensure they can always be cleared in the day-ahead electricity market. Finally, the total investment budget is set to $C^{i n v, \max }=200 \cdot 10^{6} €$. The algorithm is implemented in MATLAB and the MILP problems are solved using Gurobi 9.0.2. All simulations were performed on a desktop computer with Intel Core i $7.00 \mathrm{GHz}$ and $32 \mathrm{~GB}$ RAM.

\begin{tabular}{|c|c|c|}
\multicolumn{4}{c}{ TABLE I: Eligible Nodes for DER Installation } \\
\hline & \multicolumn{3}{|c|}{ Eligible DN Nodes } \\
& DN 1 (TN Bus \#4) & DN 2 (TN Bus \#9) \\
\hline Wind & $11,16,18,19,21,23$ & $6,25,27,29,31,32$ \\
\hline PV & $16,22,24,26,28,30$ & $1,2,7,17,20,25$ \\
\hline Storage & $5,8,16,21,22,28$ & $1,2,8,15,25,30$ \\
\hline
\end{tabular}

\section{A. Sizing and Siting Decisions}

This section describes the DER investment decisions with the rate of return that the ESP anticipates to receive from the investment being $\chi=1.15$. Figure 4 illustrates the DERs' investment decisions per node of each DN. One can see that higher DER capacity is installed in DN 2 than in DN 1. This is mainly explained by two factors: first DN 2 is
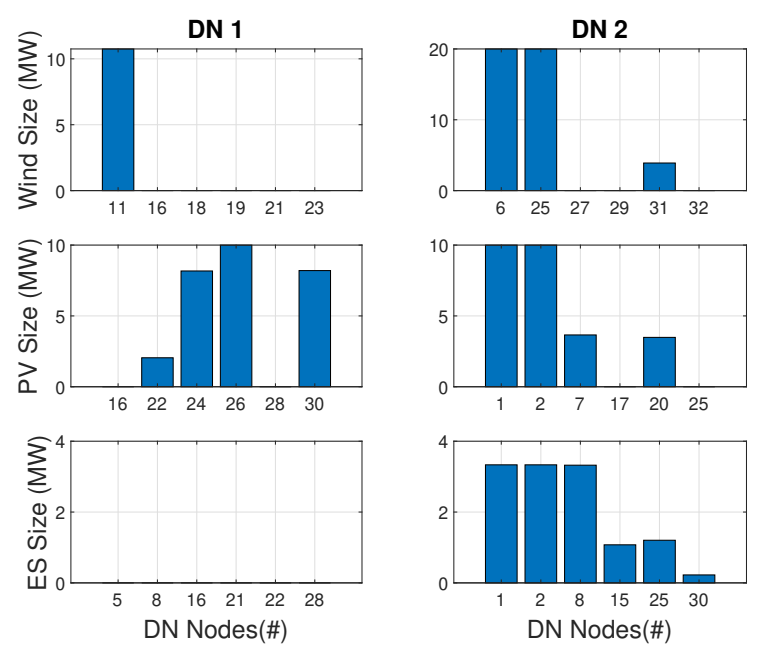

Fig. 4: DERs Sizing and Siting

located in a location (TN node \#9) with much higher wind intensity (cf. Fig. 3), and second, the available nodes for wind and PV units installation in DN 2 allow the ESP to install higher wind and solar capacity without violating the distribution network constraints. In DN 1, only solar (28MW) and wind $(10.75 \mathrm{MW})$ production units are installed, with the investment costs and the eligible nodes for solar production making the PV technology more attractive investment for the ESP than the wind production. Wind capacity is installed only at node \#11, while PV units are installed at nodes \#22, 24, 26 and 30, where either the lines connected to these nodes have enough capacity for the generated power to flow through them, or the local loads are sufficiently high in order for the net load to flow through the adjacent lines without causing over- or under-voltage issues. In DN 2, the wind intensity and the candidate DN nodes for wind capacity installation makes the wind capacity the most profitable investment in this area. Thus, a total of 44MW of wind capacity is installed together with $27 \mathrm{MW}$ of solar capacity and $75 \mathrm{MWh} / 12.5 \mathrm{MW}$ ES units. Again for reasons of high adjacent lines capacities and local loads, 10MW of wind turbines are installed at nodes \#6 and 25 , and $4 \mathrm{MW}$ at node \#31. Moreover, two PV units of 10MW each are installed very close to the DN root (nodes \#1 and 2), and two pv units of 3.5MW each at nodes \#7 and 20. In order for high wind and solar production at DN 2 to be efficiently exploited and the DN assets' payoff to be maximized, storage capacity is chosen to be installed at all eligible nodes. In more detail, the maximum possible ES capacity is installed at nodes \#1 and 2 to support the PV units installed at these nodes, and 7.2MWh/1.2MW ES units are installed at node \#25 where 20MW of wind capacity is also installed. Also, 20MWh/3.3MW, 6.40MWh/1.06MW and 1.35MWh/0.23MW of storage capacity are installed at nodes \#8, 15 and 30 to support the smooth operation of the DN and the profitability of the DN users. The DER investment decisions, which cost $124.9 \cdot 10^{6} €$, benefit all the involved actors. The ESP achieves the anticipated rate of return on its investments (115\%), while the electricity cost for DN users declines compared to the case without DER investments. The total annual DN users' electricity cost is $19.81 \cdot 10^{6} €$ without DERs, which is 
reduced by $5.45 \%$. Finally, the TSO benefits from the DER investments, since expensive TN-level electricity production is replaced in the generation mix by low-cost renewable energy units. More specifically, the TN generation cost $\left(428.07 \cdot 10^{6} €\right.$ without DERs investments) declines by $4.29 \%$.

\section{B. Impact of the ESP's minimum profit constraint}

In this subsection, we examine the impact that the choice of the rate of return $(\chi)$ has on the DER investments. To this end, we consider three different values of $\chi(1.15,1.20$ and 1.25) along with the case where the ESP's profit constraint (Eq. a.14) is not included in our model. In Fig. 5, the sizes of the DER investments are compared for the different choices of $\chi$, while in Table $\Pi$ the ESP investment costs and the $\mathrm{T} \& \mathrm{D}$ operating cost savings are presented. Without constraint

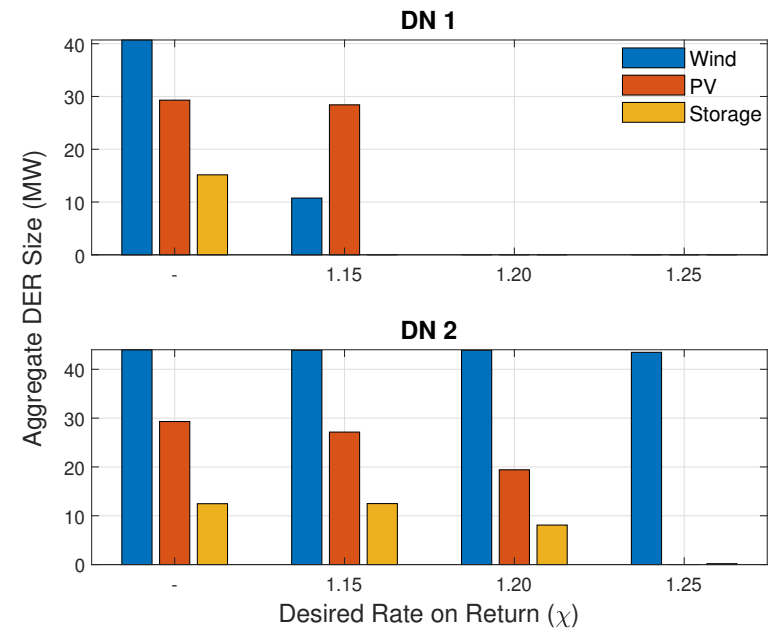

Fig. 5: DERs Investments for various values of $\chi$

a.14, the ESP installs a total of 84MW wind capacity, 58 MW solar production and $165 \mathrm{MWh} / 27.5 \mathrm{MW}$ ES capacity, which are almost evenly distributed between the two DNs, with the ESP yielding a $111 \%$ rate of return, the electricity cost of the DN consumers declining by $6.79 \%$ comparing to the case where no DERs are installed, and the transmission-level generation cost dropping by $5.87 \%$ (Table III). Incorporating constraint a.14 and as the value of $\chi$ rises, our investment model, in order for the ESP to achieve the anticipated rate of return, decides lower DER capacity, which leads in lower generation cost reduction and hence higher nodal LMPs. As a result, the energy cost reduction for the DN consumers declines and the DERs' profit increase. This is evident in Table II Increasing $\chi$ even more $(\chi=1.20$ and $\chi=1.25)$, it is in the interest of the ESP to install DERs only in DN 2 and not in DN 1, due to the increased wind intensity in this area and network characteristics as previously explained (see Section IV-A. Thus, for all values of $\chi$ under study the ESP installs 44MW of wind turbines in DN 2. In case $\chi=1.20$, it also installs $20 \mathrm{MW}$ of solar capacity, which (in order to be profitable and network feasible) is followed by investments in energy storage, as far as the latter are financially justified $(48 \mathrm{MWh} / 8 \mathrm{MW})$. When $\chi=1.25$, the only option is wind technology so as to maximize the DN assets' payoff ensuring the ESP's desired rate of return on the investments.
TABLE II: Investment Cost, Operating Cost Savings of the T\&D Systems, for Various Values of $\chi$

\begin{tabular}{|c|c|c|c|c|}
\hline$\chi$ & - & 1.15 & 1.20 & 1.25 \\
\hline $\begin{array}{c}\text { Investment Cost } \\
\left(10^{6} €\right)\end{array}$ & 175.89 & 124.90 & 78.20 & 56.64 \\
\hline $\begin{array}{c}\text { DN Users } \\
\text { Cost Savings (\%) }\end{array}$ & 6.79 & 5.45 & 5.12 & 4.24 \\
\hline $\begin{array}{c}\text { TN Generation } \\
\text { Cost Savings (\%) }\end{array}$ & 5.87 & 4.29 & 2.83 & 2.15 \\
\hline
\end{tabular}

\section{Effect of co-optimizing RES and Storage Investments}

In this subsection we consider three investment scenarios:

- Scenario 1: Only investments in storage units.

- Scenario 2: Only investments in renewable energy.

- Scenario 3: The ESP co-optimizes renewable energy and storage investments.

In Fig. 6, the DER sizes are compared for the different investment scenarios in case of not including the ESP profit constraint (Eq. (a.14)) and in case of $\chi=1.15$. In the left bar graph, where Eq. (a.14) is not included, we can see that in Scenario 3, the ESP, being entitled to invest in storage units, installs 6\% higher wind capacity and 1.2\% higher PV capacity comparing to Scenario 2. This results in $12 \%$ higher annual profits for the ESP than Scenario 2, slightly lower electricity cost for the local load and lower overall generation costs (see Table (III). Also, the installed storage capacity in Scenario 3 exceeds storage investments in Scenario 1 by $25 \%$, since this enables larger RES capacity installation and ultimately higher aggregate DN users market gain. In Table III] one can see that investing solely in storage assets, not taking into consideration the ESP's return on investments, which is the case in [19], the optimal investment decisions achieve a $4.42 \%$ energy cost reduction for end users. However, the ESP does not cover its capital costs losing 0.4 million euros annually. In case of
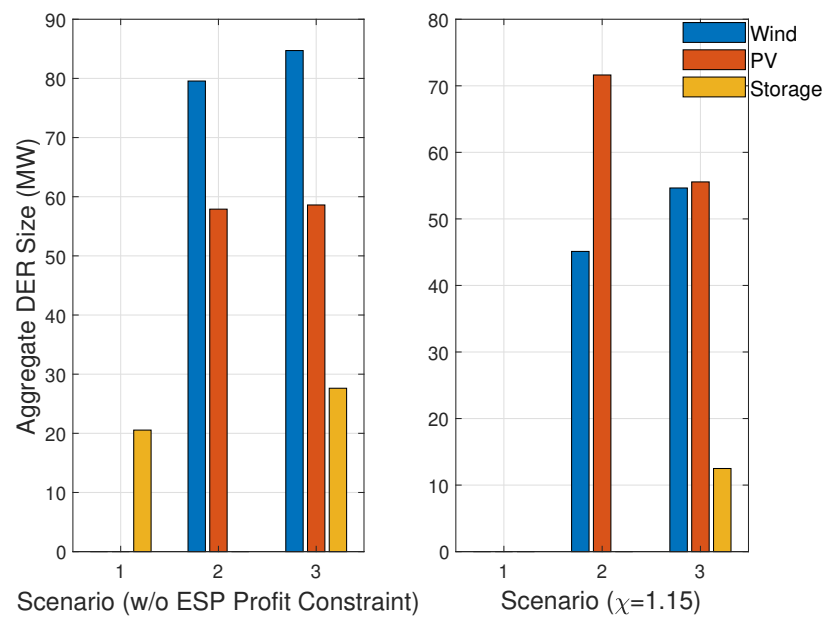

Fig. 6: DERs Investments in different investment scenarios

$\chi=1.15$ (right bar graph), in Scenario 1 the ESP does not invest in energy storage at all, since the storage capital costs, the market prices, the eligible nodes for storage investments and the distribution network limitations do not enable the ESP to make adequate market profits so as to reach the desired rate of return on its investments. As it is also evident in Section IV-B, incorporating the ESP's profit constraint, investments 
in storage increasingly decline with increasing $\chi$. Comparing investment scenarios 2 and 3 when $\chi=1.15$, we can see in Fig. 6 that co-optimizing RES and storage investments, the ESP achieves $15 \%$ profit by installing $5.5 \%$ less renewable energy capacity comparing to the case in which the ESP installs only renewable energy capacity. The ESP in Scenario 3 achieves 6\% higher annual net profits comparing to Scenario 2, while co-optimizing RES and ES investments, the generation cost reduction and the DN consumers energy cost reduction is slightly larger than in the case where only RES units are considered.

TABLE III: Investment Cost, Operating Cost Savings of the T\&D Systems, and ESP Market Profits in Different Investment Scenarios

\begin{tabular}{|c|c|c|c|c|c|c|}
\hline$\chi$ & \multicolumn{3}{|c|}{-} & \multicolumn{3}{|c|}{1.15} \\
\hline Scenario & 1 & 2 & 3 & 1 & 2 & 3 \\
\hline $\begin{array}{c}\text { Investment Cost } \\
\left(10^{6} €\right)\end{array}$ & 12.74 & 151.48 & 175.89 & 0 & 118.10 & 124.90 \\
\hline $\begin{array}{c}\text { DN Users } \\
\text { Cost Savings (\%) }\end{array}$ & 4.42 & 6.66 & 6.79 & 0 & 5.40 & 5.45 \\
\hline $\begin{array}{c}\text { TN Generation } \\
\text { Cost Savings (\%) }\end{array}$ & 0.31 & 5.13 & 5.87 & 0 & 4.07 & 4.29 \\
\hline $\begin{array}{c}\text { ESP Annualized } \\
\text { Clear Profits } \\
\left(10^{6} €\right)\end{array}$ & -0.40 & 2.06 & 2.31 & 0 & 2.07 & 2.19 \\
\hline
\end{tabular}

\section{Bi-level Vs. Single-level Modeling}

This subsection compares our bi-level model to a singlelevel investment model setting $\chi=1.25$. In the latter case, only problem (a) is solved with market prices being input parameters based on price forecasts (as in [20]- [25]). The price forecasts are produced solving problem (b) for each representative day considering no DER investments. Hence, the impact of the DER investments on energy market price is not taken into consideration, resulting in over-investments that hamper the ESP's return on investment.

More specifically, solving the single-level model, $85 \mathrm{MW}$ of wind capacity, $7.5 \mathrm{MW}$ of PV capacity and a total of $7 \mathrm{MWh} / 1.16 \mathrm{MW}$ storage capacity are installed. These investments, with a total investment cost of $118 \cdot 10^{6} €$ are calculated based on the assumed market prices and seemingly achieve the desired rate of return on DER investments. However, the installed DERs lead in market price changes, which eventually produce lower market profits for the ESP than the ones that had been calculated solving the single-level model. The T\&D operating cost reduction is higher in case of the single-level model (see Table IV), since larger DER capacities achieve lower LMPs at the TSO-DSO coupling points, but on the other hand the resulting rate of return is insufficient for the ESP.

TABLE IV: Investment Cost, Operating Cost Savings of the T\&D Systems, and Rate of Return on ESP Investments in Single-level and Bi-level Investment Models

\begin{tabular}{|c|c|c|}
\hline & Single-Level Model & Bi-Level Model \\
\hline $\begin{array}{c}\text { Investment Cost } \\
\left(10^{6} €\right)\end{array}$ & 118.36 & 56.64 \\
\hline $\begin{array}{c}\text { DN Users } \\
\text { Cost Savings (\%) }\end{array}$ & 6.33 & 4.24 \\
\hline $\begin{array}{c}\text { TN Generation } \\
\text { cost Savings (\%) }\end{array}$ & 4.10 & 2.15 \\
\hline Rate of Return (\%) & 113 & 130 \\
\hline
\end{tabular}

\section{E. Computational Efficiency}

We test the computational efficiency of our proposed nested decomposition algorithm using the IEEE 118-bus system [39] and the IEEE 33-Node Distribution Test System. Towards this goal, we compare our algorithm to the non-decomposed MILP (which results from converting the bi-level problem (a)-(b) through an MPEC problem as also explained in the beginning of Section III) for various numbers of representative days $(|\Omega|)$ in terms of solution time.

As we can see in Table $\mathrm{V}$, in case $|\Omega|=4$, the nondecomposed MILP (ND-MILP) achieves the optimal solution much faster than our proposed solution method, which reaches a sub-optimal solution by $0.42 \%$. However, the computational burden increases with the number of representative days $|\Omega|$. Thus, the execution time for the non-decomposed MILP upsurges for $|\Omega|=8$, while for $|\Omega|=16$ and $|\Omega|=24$, the non-decomposed MILP cannot find a feasible integer solution within the predefined time limit (48h or $172,800 \mathrm{sec}$ ). On the other hand, our proposed nested decomposition algorithm reaches the optimal solution in 4 or 5 outer decomposition iterations. The average number of inner decomposition iterations reduces with $|\Omega|$, since as also stated in Section III-B the objective function of the problem convexifies with respect to the investment variables as $|\Omega|$ increases. Since the functioning of outer and inner decomposition procedures improves with $|\Omega|$ (as explained in Section III), the optimality loss also declines with increasing number of representative days. Conclusively, our proposed algorithm reaches an optimal solution within a finite number of iterations, without sacrificing optimality, significantly reducing the computational burden that results in intractability issues for the non-decomposed MILP.

\begin{tabular}{|l|c|c|c|c|c|c|}
\multicolumn{7}{c|}{ TABLE V: Computational Performance } \\
\begin{tabular}{|l|c|c|c|c|c|} 
Solution \\
Method
\end{tabular} & $|\Omega|$ & $\begin{array}{c}\text { Outer } \\
\text { Iter. }\end{array}$ & $\begin{array}{c}\text { Inner } \\
\text { Iter. }\end{array}$ & $\begin{array}{c}\text { Exec. } \\
\text { Time (s) }\end{array}$ & $\begin{array}{c}\text { Opt. } \\
\text { Result }\end{array}$ & $\begin{array}{c}\text { Opt. } \\
\text { Loss (\%) }\end{array}$ \\
\hline \multirow{3}{*}{ ND- } & 4 & - & - & 637 & 73826 & - \\
MILP & 8 & - & - & 10079 & 76287 & - \\
& 16 & - & - & 172800 & - & - \\
& 24 & - & - & 172800 & - & - \\
\hline & 4 & 4 & 55 & 11594 & 73517 & 0.42 \\
Proposed & 8 & 5 & 38 & 15143 & 76242 & 0.06 \\
Method & 16 & 5 & 33 & 28375 & 74020 & - \\
& 24 & 5 & 29 & 43814 & 75605 & - \\
\hline
\end{tabular}

\section{CONCLusion}

In this paper, we model the DER investment problem of a private ESP, which installs distributed renewable energy and energy storage units in multiple DNs within a TSO-DSO coordination scheme. The rate of return on the DER investment is ensured. A stochastic bi-level investment model is formulated, which is efficiently solved using a nested decomposition algorithm based on the concepts of Bender's decomposition and Lagrangian relaxation. Our algorithm calculates the DER sizing and sitting decisions in a finite number of iterations without sacrificing optimality. The proposed framework can be used by a regulator or policy making entity to efficiently coordinate the business interests of ESP, DSO and TSO to facilitate a quicker renewable energy transition. As a future work, we plan to study the coordination of DER investments and distribution network expansion. Also, our study can be 
extended taking into account more revenue streams for the DERs (e.g. ancillary services provision to both the TSO and the DSO).

\section{APPENDIX}

\section{A. Transforming bi-level problem (c) into a MILP}

Lower-level problem (b) is an LP and therefore, Slater's condition holds. Thus, Karush-Kuhn-Tucker (KKT) conditions are necessary and sufficient optimality conditions (satisfy convexity and constraint qualification). The KKT conditions of problem (b) are presented below:

$$
-p_{i t \omega}^{g}+p_{i t \omega}^{d}-p_{i t \omega}^{\uparrow}+p_{i t \omega}^{\downarrow}+\sum_{j \neq i} y_{i j} \cdot\left(\theta_{i t \omega}-\theta_{j t \omega}\right)=0
$$

$\forall i \in N, t \in H$

$c_{i t}^{g}-\lambda_{i t \omega}-\underline{\phi_{i t \omega}^{g}}+\overline{\phi_{i t \omega}^{g}}-\phi_{i t \omega}^{g r d}+\phi_{i(t+1) \omega}^{g r d}+\phi_{i t \omega}^{g r u}-\phi_{i(t+1) \omega}^{g r u}$ $=0, \quad \forall i \in N^{g}, t<T$

$c_{i t}^{g}-\lambda_{i t \omega}-\underline{\phi_{i t \omega}^{g}}+\overline{\phi_{i t \omega}^{g}}-\phi_{i t \omega}^{g r d}+\phi_{i t \omega}^{g r u}=0$,

$\forall i \in N^{g}, t=T$

$-c_{i t}^{d}+\lambda_{i t \omega}-\underline{\phi_{i t \omega}^{d}}+\overline{\phi_{i t \omega}^{d}}=0, \quad \forall i \in N^{d}, t \in H$

$c_{i t}^{\uparrow}-\lambda_{i t \omega}-\underline{\phi_{i t \omega}^{p \uparrow}}+\overline{\phi_{i t \omega}^{p \uparrow}}=0, \quad \forall i \in N^{m}, t \in H$

$-c_{i t}^{\downarrow}+\lambda_{i t \omega}-\underline{\phi_{i t \omega}^{p \downarrow}}+\overline{\phi_{i t \omega}^{p \downarrow}}=0, \quad \forall i \in N^{m}, t \in H$

$\left.\sum_{j \neq i,(i, j) \in L^{T}} y_{i j} \cdot\left(\lambda_{i t \omega}-\lambda_{j t \omega}\right)-\sum_{j>i} y_{j i} \cdot \underline{\left(\phi_{(i j) t \omega}^{l}\right.}-\overline{\phi_{(i j) t \omega}^{l}}\right)$

$\left.+\sum_{j<i} y_{j i} \cdot \underline{\left(\phi_{(i j) t \omega}^{l}\right.}-\overline{\phi_{(i j) t \omega}^{l}}\right)=0, \quad \forall i \in N, t \in H$

$0 \leq \underline{\phi_{i t \omega}^{g}} \perp p_{i t \omega}^{g} \geq 0, \quad \forall i \in N^{g}, t \in H$

$0 \leq \overline{\overline{\phi_{i t \omega}^{g}}} \perp-p_{i t \omega}^{g}+\overline{P_{i}^{g}} \geq 0, \quad \forall i \in N^{g}, t \in H$

$0 \leq \phi_{i t \omega}^{g r d} \perp p_{i t \omega}^{g}-p_{i(t-1) \omega}^{g}+R D_{i} \geq 0, \quad \forall i \in N^{g}, t \in H$

$0 \leq \phi_{i t \omega}^{g r u} \perp-p_{i t \omega}^{g}+p_{i(t-1) \omega}^{g}+R U_{i} \geq 0, \quad \forall i \in N^{g}, t \in H$

$0 \leq \underline{\phi_{i t \omega}^{d}} \perp p_{i t \omega}^{d} \geq 0, \quad \forall i \in N^{d}, t \in H$

$0 \leq \overline{\overline{\phi_{i t \omega}^{d}}} \perp-p_{i t \omega}^{d}+\overline{P_{i t \omega}^{d}} \geq 0, \quad \forall i \in N^{d}, t \in H$

$0 \leq \underline{\phi_{i t \omega}^{p \uparrow}} \perp p_{i t \omega}^{\uparrow} \geq 0, \quad \forall i \in N^{m}, t \in H$

$0 \leq \overline{\phi_{i t \omega}^{p \uparrow}} \perp-p_{i t \omega}^{\uparrow}+o_{i t \omega} \geq 0, \quad \forall i \in N^{m}, t \in H$

$0 \leq \underline{\phi_{i t \omega}^{p \downarrow}} \perp p_{i t \omega}^{\downarrow} \geq 0, \quad \forall i \in N^{m}, t \in H$

$0 \leq \overline{\phi_{i t \omega}^{p \downarrow}} \perp-p_{i t \omega}^{\downarrow}+b_{i t \omega} \geq 0, \quad \forall i \in N^{m}, t \in H$

$0 \leq \phi_{(i j) t \omega}^{l} \perp y_{i j} \cdot\left(\theta_{i t \omega}-\theta_{j t \omega}\right)+\overline{T_{i j}} \geq 0$,

$\forall(i, j) \in L, t \in H$

$0 \leq \overline{\phi_{(i j) t \omega}^{l}} \perp-y_{i j} \cdot\left(\theta_{i t \omega}-\theta_{j t \omega}\right)+\overline{T_{i j}} \geq 0$,

$\forall(i, j) \in L, t \in H$

Equation h.1 is the equality constraint of the Lower-Level problem, while Eqs. (h.2)-(h.7) are the stationarity conditions. Finally, (h.8)-(h.19) are the complementarity slackness conditions. We use the perpendicular symbol $(\perp)$ to indicate complementarity. Replacing the constraining problem (b) with its KKT conditions results in the following MPEC problem:

$$
\begin{array}{r}
\min _{X_{S}^{U} \cup X_{O, \omega}^{U} \cup X_{\omega}^{L}, \forall \omega} \sum_{\omega \in \Omega}\left(-P r_{\omega}^{D N}+C_{\omega}^{\text {oper }}\right)+\tilde{C}^{i n v} \\
+\xi \cdot\left(\sum_{\omega \in \Omega}\left(-P r_{\omega}^{i n v}+C_{\omega}^{\text {oper }}\right)+\chi \cdot \tilde{C}^{i n v}\right)
\end{array}
$$

Subject to

$$
a .5-a .13, a .16-a .31, h .1-h .19
$$

Problem (i) is a single-level MINLP. The nonlinearities due to the complementarity conditions are linearized using the Big$\mathrm{M}$ approach [40]. Complementarity constraints of the type $0 \leq \alpha \perp \beta \geq 0$ are replaced by the following set of linear constraints:

$$
\begin{aligned}
& 0 \leq \alpha \leq M \cdot u \\
& 0 \leq \beta \leq M \cdot(1-u)
\end{aligned}
$$

where $M$ is a large constant and $u$ is an auxiliary binary variable. In order to tackle the nonlinearities in the objective function concerning expression in a.2, we first multiply Eqs. (h.5) and h.6 by $p_{i t \omega}^{\uparrow}$ and $p_{i t \omega}^{\downarrow}$ respectively:

$$
\begin{aligned}
& c_{i t}^{\uparrow} \cdot p_{i t \omega}^{\uparrow}-\lambda_{i t \omega} \cdot p_{i t \omega}^{\uparrow}-\underline{\phi_{i t \omega}^{p \uparrow}} \cdot p_{i t \omega}^{\uparrow}+\overline{\phi_{i t \omega}^{p \uparrow}} \cdot p_{i t \omega}^{\uparrow}=0, \\
& \forall i \in N^{m}, t \in H \\
& -c_{i t}^{\downarrow} \perp p_{i t \omega}^{\downarrow}+\lambda_{i t \omega} \perp p_{i t \omega}^{\downarrow}-\underline{\phi_{i t \omega}^{p \downarrow}} \perp p_{i t \omega}^{\downarrow}+\overline{\phi_{i t \omega}^{p \downarrow}} \perp p_{i t \omega}^{\downarrow}=0, \\
& \forall i \in N^{m}, t \in H
\end{aligned}
$$

Then, using the complementarity conditions (h.14)-(h.17) and re-arranging terms, we have:

$$
\begin{aligned}
& \sum_{i \in N^{m}} \sum_{t \in H}\left(\lambda_{i t \omega} \cdot\left(p_{i t \omega}^{\uparrow}-p_{i t \omega}^{\downarrow}\right)\right)= \\
& \sum_{i \in N^{m}} \sum_{t \in H}\left(c_{i t}^{\uparrow} \cdot p_{i t \omega}^{\uparrow}-c_{i t}^{\downarrow} \cdot p_{i t \omega}^{\downarrow}+\overline{\phi_{i t \omega}^{p \uparrow}} \cdot \dot{i t \omega}+\overline{\phi_{i t \omega}^{p \downarrow}} \cdot b_{i t \omega}\right.
\end{aligned}
$$

Now, we make use of the Strong Duality Theorem for problem (b), which states:

$$
\begin{aligned}
& \sum_{t \in H}\left(\sum_{i \in N^{g}} c_{i t}^{g} \cdot p_{i t \omega}^{g}-\sum_{i \in N^{d}} c_{i t}^{d} \cdot p_{i t \omega}^{d}+\sum_{i \in N^{m}}\left(c_{i t}^{\uparrow} \cdot p_{i t \omega}^{\uparrow}-\right.\right. \\
& \left.\left.c_{i t}^{\downarrow} \cdot p_{i t \omega}^{\downarrow}\right)\right)=-\sum_{t \in H}\left(\sum _ { i \in N ^ { g } } \left(\overline{\phi_{i t \omega}^{g}} \cdot \overline{P_{i}^{g}}+\phi_{i t \omega}^{g r d} \cdot R D_{i}+\phi_{i t \omega}^{g r u} \cdot\right.\right. \\
& \left.\left.R U_{i}\right)+\sum_{i \in N^{d}}\left(\overline{\phi_{i t \omega}^{d}} \cdot \overline{P_{i t \omega}^{d}}\right)+\sum_{i \in N^{m}} \overline{\left(\phi_{i t \omega}^{p \uparrow}\right.} \cdot o_{i t \omega}+\overline{\phi_{i t \omega}^{p \downarrow}} \cdot b_{i t \omega}\right)+ \\
& \left.+\sum_{i<j,(i, j) \in L}\left(\overline{T_{i j}} \cdot \underline{\phi_{(i j) t \omega}^{l}}+\overline{T_{i j}} \cdot \overline{\phi_{(i j) t \omega}^{l}}\right)\right)
\end{aligned}
$$

Re-arranging the terms in the above expression, we obtain:

$$
\begin{aligned}
& \sum_{t \in H}\left(\sum_{i \in N^{m}}\left(c_{i t}^{\uparrow} \cdot p_{i t \omega}^{\uparrow}-c_{i t}^{\downarrow} \cdot p_{i t \omega}^{\downarrow}+\overline{\phi_{i t \omega}^{p \uparrow}} \cdot o_{i t \omega}+\overline{\phi_{i t \omega}^{p \downarrow}} \cdot b_{i t \omega}\right)\right. \\
& =-\sum_{t \in H}\left(\sum _ { i \in N ^ { g } } \left(c_{i t}^{g} \cdot p_{i t \omega}^{g}+\overline{\phi_{i t \omega}^{g}} \cdot \overline{P_{i}^{g}}+\phi_{i t \omega}^{g r d} \cdot R D_{i}+\phi_{i t \omega}^{g r u}\right.\right. \\
& \left.\cdot R U_{i}\right)+\sum_{i \in N^{d}}\left(-c_{i t}^{d} \cdot p_{i t \omega}^{d}+\overline{\phi_{i t \omega}^{d}} \cdot \overline{P_{i t \omega}^{d}}\right)+
\end{aligned}
$$


$\sum_{i<j,(i, j) \in L}\left(\overline{T_{i j}} \cdot \underline{\phi_{(i j) t \omega}^{l}}+\overline{T_{i j}} \cdot \overline{\phi_{(i j) t \omega}^{l}}\right)$

Hence, nonlinear expression a.2 is replaced by its linear equivalent expression:

$$
\begin{aligned}
& \operatorname{Pr}_{\omega}^{D N}=-\pi_{\omega} \cdot \sum_{t \in H}\left(\sum _ { i \in N ^ { g } } \left(c_{i t}^{g} \cdot p_{i t \omega}^{g}+\overline{\phi_{i t \omega}^{g}} \cdot \overline{P_{i}^{g}}+\phi_{i t \omega}^{g r d} \cdot R D_{i}\right.\right. \\
& \left.+\phi_{i t \omega}^{g r u} \cdot R U_{i}\right)+\sum_{i \in N^{d}}\left(-c_{i t}^{d} \cdot p_{i t \omega}^{d}+\overline{\phi_{i t \omega}^{d}} \cdot \overline{P_{i t \omega}^{d}}\right)+ \\
& \sum_{i<j,(i, j) \in L}\left(\overline{T_{i j}} \cdot \underline{\phi_{(i j) t \omega}^{l}}+\overline{T_{i j}} \cdot \overline{\phi_{(i j) t \omega}^{l}}\right)
\end{aligned}
$$

Finally, in order to linearize the expression in a.15), since we have considered a lossless DN model, we use the active power balance equations of the DN (i.e. (a.26) and (a.31p):

$$
\begin{aligned}
& \pi_{\omega} \cdot \sum_{i \in N^{m}} \sum_{t \in H}\left(\lambda _ { i t \omega } \cdot \left(\sum_{n \in B_{i}^{w}} g_{i n t \omega}^{w}+\sum_{n \in B_{i}^{p v}} g_{i n t \omega}^{p v}+\right.\right. \\
& \left.\left.\sum_{n \in B_{i}^{e s}}\left(d i s_{\text {int } \omega}-c h_{\text {int } \omega}\right)\right)\right)= \\
& \pi_{\omega} \cdot \sum_{i \in N^{m}} \sum_{t \in H}\left(\lambda_{i t \omega} \cdot\left(\left(p_{i t \omega}^{\uparrow}-p_{i t \omega}^{\downarrow}\right)+\sum_{n \in B} D_{n t \omega}\right)\right)
\end{aligned}
$$

Then the remaining nonlinear term $\lambda_{i t \omega} \cdot\left(p_{i t \omega}^{\uparrow}-p_{i t \omega}^{\downarrow}\right)$ is linearized as previously mentioned. Thus, we have transformed the bi-level problem (c) into a MILP.

\section{B. Formulation of $S P 1$}

We transform the bi-level problem (f) into a MILP using the MPEC method. We replace the lower-level problem (b) with its KKT conditions. Hence, problem (f) can be recast into the following MINLP problem:

$$
\min _{X_{O, \omega}^{U} \cup X_{\omega}^{L}} \tilde{G}_{\omega}=(1+\xi) \cdot C_{\omega}^{o p e r}-P r_{\omega}^{D N}-\xi \cdot P r_{\omega}^{i n v}
$$

Subject to

$$
\text { a.5, a.6, a.16 - a.31, } h .1-a .19,6 f .3-f .6
$$

The above MINLP is transformed into a MILP as explained in Section V-A. which can be solved using off-the-shelf solvers. The optimal values $h_{i t \omega}^{*}, x_{i n t \omega}^{*}, \overline{\phi_{i t \omega}^{p \uparrow *}}, \phi_{i t \omega}^{p \uparrow *}, \overline{\phi_{i t \omega}^{p \downarrow *}}, \phi_{i t \omega}^{p \downarrow *}$ provided by the solution of problem (j) will be used in the formulation of SP2 (cf Section V-C).

\section{Formulation of $S P 2$}

Now, we transform the bi-level problem (f) into an LP using the MPPDC method. We replace the lower-level problem (b) with its primal constraints, its dual constraints and the Strong Duality Theorem expression. Also, we relax the integrality conditions (a.18) and (a.22), and we fix the values of $h_{i t \omega}$ and $x_{\text {int }}$ to their optimal values calculated in SP1. Furthermore, bilinear terms in the Strong Duality Theorem expression are linearized replacing dual variables $\phi_{i t \omega}^{p \uparrow}, \phi_{i t \omega}^{p \downarrow}$ with their respective optimal values calculated solving SP1 (i.e. $\overline{\phi_{i t \omega}^{p \uparrow *},}, \phi_{i t \omega}^{p \downarrow \text { ) }}$ ). Therefore, problem (f) is converted into the following NLP:

$$
\min _{X_{O, \omega}^{U} \cup X_{\omega}^{L}} \tilde{G}_{\omega}=(1+\xi) \cdot C_{\omega}^{o p e r}-\operatorname{Pr}_{\omega}^{D N}-\xi \cdot \operatorname{Pr}_{\omega}^{i n v}
$$

Subject to

a.5, a.6, a.16, a.17, a.19 - a.21, a.23 - a.31

b.2. $-b .9$

$\underline{\phi_{i t \omega}^{g}}, \overline{\phi_{i t \omega}^{g}}, \phi_{i t \omega}^{g r d}, \phi_{i t \omega}^{g r u}, \underline{\phi_{i t \omega}^{d}}, \overline{\phi_{i t \omega}^{d}}, \underline{\phi_{i t \omega}^{p \uparrow}}, \overline{\phi_{i t \omega}^{p \uparrow}}, \underline{\phi_{i t \omega}^{p \downarrow}}, \overline{\phi_{i t \omega}^{p \downarrow}}$,

$\underline{\phi_{(i j) t \omega}^{l},}, \overline{\phi_{(i j) t \omega}^{l}} \geq 0$

h.2. $-h .7$

$\sum_{t \in H}\left(\sum_{i \in N^{g}} c_{i t}^{g} \cdot p_{i t \omega}^{g}-\sum_{i \in N^{d}} c_{i t}^{d} \cdot p_{i t \omega}^{d}+\sum_{i \in N^{m}}\left(c_{i t}^{\uparrow} \cdot p_{i t \omega}^{\uparrow}-\right.\right.$

$\left.\left.c_{i t}^{\downarrow} \cdot p_{i t \omega}^{\downarrow}\right)\right)=-\sum_{t \in H}\left(\sum_{i \in N^{g}}\left(\overline{\phi_{i t \omega}^{g}} \cdot \overline{P_{i}^{g}}+\phi_{i t \omega}^{g r d} \cdot R D_{i}+\phi_{i t \omega}^{g r u}\right.\right.$.

$\left.R U_{i}\right)+\sum_{i \in N^{d}}\left(\overline{\phi_{i t \omega}^{d}} \cdot \overline{P_{i t \omega}^{d}}\right)+\sum_{i \in N^{m}}\left(\overline{\phi_{i t \omega}^{p \uparrow *}} \cdot o_{i t \omega}+\overline{\phi_{i t \omega}^{p \downarrow *}} \cdot b_{i t \omega}\right)+$

$\left.+\sum_{i<j,(i, j) \in L}\left(\overline{T_{i j}} \cdot \underline{\phi_{(i j) t \omega}^{l}}+\overline{T_{i j}} \cdot \overline{\phi_{(i j) t \omega}^{l}}\right)\right)$

$f .3-f .6$

$h_{i t \omega}=h_{i t \omega}^{*}$

$x_{i n t \omega}=x_{i n t \omega}^{*}$

$\underline{\phi_{i t \omega}^{p \uparrow}}=\underline{\phi_{i t \omega}^{p \uparrow *}}$

$\overline{\phi_{i t \omega}^{p \uparrow}}=\overline{\phi_{i t \omega}^{p \uparrow *}}$

$\frac{\phi_{i t \omega}^{p \downarrow}}{\overline{\phi_{i t \omega}^{p \downarrow}}}=\frac{\phi_{i t \omega}^{p \downarrow *}}{\overline{\phi_{i t \omega}^{p \downarrow *}}}$

The above problem $(\mathrm{k})$ is a continuous non-linear optimization problem. Non-linearities in the objective function (k.1) are linearized as explained in Section $\mathrm{V}-\mathrm{B}$ and eventually problem (f) is converted in an LP.

\section{REFERENCES}

[1] FERC Order 2222,"Recommendation for the U.S. Department of Energy - Outline", April 2021. [Online]. Available: https://www.energy.gov/sites/default/files/2021-04/EAC\%20FERC\% 20Order\%202222\%20Recommendations\%202021-04-15_finalDraft.pdf

[2] DIRECTIVE (EU) 2019/944 OF THE EUROPEAN PARLIAMENT AND OF THE COUNCIL of 5 June 2019 on common rules for the internal market for electricity and amending Directive 2012/27/EU. [Online]. Available: https://eur-lex.europa.eu/legal-content/EN/TXT/?uri= CELEX\%3A32019L0944

[3] International Energy Agency, "China Power System Transformation: Assessing the benefit of optimised operations and advanced flexibility options", OECD Publishing, Paris, March 2019. [Online]. Available: https://doi.org/10.1787/22655cc0-en

[4] L. Baringo and A. J. Conejo, "Wind Power Investment: A Benders Decomposition Approach," IEEE Transactions on Power Systems, vol. 27, no. 1, pp. 433-441, Feb. 2012.

[5] E. Nasrolahpour, S. J. Kazempour, H. Zareipour, and W. D. Rosehart, "Strategic Sizing of Energy Storage Facilities in Electricity Markets", IEEE Transactions on Sustainable Energy, vol. 7, no. 4, pp. 1462-1472, Oct. 2016.

[6] D. Chen, Z. Jing, and H. Tan, "Optimal Siting and Sizing of Used Battery Energy Storage Based on Accelerating Benders Decomposition", IEEE Access, vol. 7, pp. 42993-43003, Mar. 2019.

[7] B. Xu, Y. Wang, Y. Dvorkin, R. Fenrandez-Blanco, A. Silva-Monroy, J.-P. Watson, and D. S. Kirschen, "Scalable Planning for Energy Storage in Energy and Reserve Markets", IEEE Transactions on Power Systems, vol. 32, no. 6, pp. 4515-4527, Nov. 2017. 
[8] H. Saber, H. Heidarabadi, M. Moeini-Aghtaie, H. Farzin, and M. R. Karimi, "Expansion Planning Studies of Independent-Locally Operated Battery Energy Storage Systems (BESSs): A CVaR-Based Study", IEEE Transactions on Sustainable Energy, vol. 11, no. 4, pp. 2109-2118, Oct. 2020.

[9] Y. Dvorkin, R. Fernandez-Blanco, D. S. Kirschen, H. Pandzic, J.-P. Watson, and C. A. Silva-Monroy, "Ensuring Profitability of Energy Storage", IEEE Transactions on Power Systems, vol. 32, no. 1, pp. 611623, Jan. 2017.

[10] H. Pandzic, Y. Dvorkin, and M. Carrion, "Investments in merchant energy storage: Trading-off between energy and reserve markets", Applied Energy, vol. 230, pp. 277-286, 2018.

[11] E. Mortaz, "Portfolio Diversification for an Intermediary Energy Storage Merchant", IEEE Transactions on Sustainable Energy, vol. 11, no. 3, pp. 1539-1547, Jul. 2020.

[12] N. Covic, F. Braeuer, R. Mckenna, and H. Pandzic, "Optimal PV and Battery Investment of Market-Participating Industry Facilities", IEEE Transactions on Power Systems, vol. 36, no. 4, pp. 3441-3452, Jul. 2021.

[13] M. Zidar, P. S. Georgilakis, N.D.Hatziargyriou, T. Capuder, and D. Skrlec, "Review of energy storage allocation in power distribution networks: Applications, methods and future research", IET Generation, Transmission \& Distribution, vol. 10, no. 3, pp. 645-652, Feb. 2016.

[14] H. Pandžić, Y. Wang, T. Qiu, Y. Dvorkin and D. S. Kirschen, "NearOptimal Method for Siting and Sizing of Distributed Storage in a Transmission Network", IEEE Transactions on Power Systems, vol. 30 no. 5, pp. 2288-2300, Sep. 2015.

[15] H. Gerard, E. I. R. Puente and D. Six, "Coordination between transmission and distribution system operators in the electricity sector: A conceptual framework", Elsevier Utilities Policy, vol. 50, pp. 40- 48, 2018.

[16] H. L. Cadre, I. Mezghani and A. Papavasiliou, "A game-theoretic analysis of transmission-distribution system operator coordination", Elsevier European Journal of Operational Research, vol. 274, no. 1, pp. 317-339, 2019.

[17] Z. Li, Q. Guo, H. Sun, and J. Wang, "Coordinated economic dispatch of coupled transmission and distribution systems using heterogeneous decomposition", IEEE Transactions on Power Systems, vol. 31, no. 6, pp. 4817-4830, Nov. 2016.

[18] Z. Li, Q. Guo, H. Sun, and J. Wang, "A new LMP-sensitivity-based heterogeneous decomposition for transmission and distribution coordinated economic dispatch", IEEE Transactions on Smart Grid, vol. 9, no. 2, pp. 931-941, Mar. 2018.

[19] A. Hassan, and Y. Dvorkin, "Energy Storage Siting and Sizing in Coordinated Distribution and Transmission Systems", IEEE Transactions on Sustainable Energy, vol. 9, no. 4, pp. 1692-1701, Oct. 2018.

[20] M. Moradijoz, M. P. Moghaddam, and M.-R. Haghifam, "A Flexibile Distribution System Expansion Planning Model: A Dynamic Bi-Level Approach", IEEE Transactions on Smart Grid, vol. 9, no. 6, pp. 58675877, Nov. 2018

[21] M. Mozaffari, H. A. Abyaneh, M. Jooshaki, and M. Moeini-Aghtaie, "Joint Expansion Planning Studies of EV Parking Lots Placement and Distribution Network", IEEE Transactions on Industrial Informatics, vol. 16 , no. 10 , pp. $6455-6465$, Oct. 2020

[22] J. Kim and Y. Dvorkin, "Enhancing Distribution System Resilience With Mobile Energy Storage and Microgrids," IEEE Transactions on Smart Grid, vol. 10, no. 5, pp. 4996-5006, Sep. 2019.

[23] E. G. Silva, R. Girard, G. Kariniotakis, "Optimal sizing and placement of distribution grid connected battery systems through an SOCP optimal power flow algorithm", Applied Energy, 219, pp.385-393, 2018.

[24] R. Li, W. Wang, Z. Chen and X. Wu, "Optimal planning of energy storage system in active distribution system based on fuzzy multiobjective bi-level optimization," Journal of Modern Power Systems and Clean Energy, vol. 6, no. 2, pp. 342-355, Mar. 2018.

[25] J. Xiao, Z. Q. Zhang, L. Q. Bai, and H. S. Liang, "Determination of the optimal installation site and capacity of battery energy storage system in distribution network integrated with distributed generation," IET Generation, Transmission \& Distribution, vol. 10, no. 3, pp. 601607, Feb. 2016

[26] X. Xu, J. Li, Y. Xu, Z. Xu, and C. S. Lai, ”A Two-Stage Game-Theoretic Method for Residential PV Panels Planning Considering Energy Sharing Mechanism", IEEE Transactions on Power Systems, vol. 35, no. 5, pp. 3562-3573, Sep. 2020.

[27] M. E. Baran, F. F. Wu, "Network reconfiguration in distribution systems for loss reduction and load balancing", IEEE Transactions on Power Delivery, vol. 4, no. 2, pp. 1401-1407, Apr. 1989.

[28] K. Steriotis, K. Šepetanc, K. Smpoukis, N. Efthymiopoulos, P. Makris, E. Varvarigos, and H. Pandžić, "Stacked Revenues Maximization of
Distributed Battery Storage Units via Emerging Flexibility Markets", IEEE Transactions on Sustainable Energy, Early Access.

[29] Y. Dvorkin, "Can Merchant Demand Response Affect Investments in Merchant Energy Storage?", IEEE Transactions on Power Systems, vol. 33, no. 3, pp. 2671-2683, May 2018.

[30] L. Baringo, and A. J. Conejo, "Strategic Wind Power Investment", IEEE Transactions on Power Systems, vol. 29, no. 3, pp. 1250-1260, May 2014.

[31] M. L. Fisher, "An applications oriented guide to Lagrangian relaxation", Interfaces, vol. 15, no. 2, pp. 10-21, 1985.

[32] A. J. Conejo, E. Castillo, R. Minguez, and R. Garcia-Bertrand, 'Decomposition Techniques in Mathematical Programming: Engineering and Science Applications", USA, NY, New York: Springer, 2006.

[33] K. Steriotis, K. Smpoukis, N. Efthymiopoulos, G. Tsaousoglou, P. Makris, E. Varvarigos, "Strategic and network-aware bidding policy for electric utilities through the optimal orchestration of a virtual and heterogeneous flexibility assets' portfolio", Electric Power Systems Research, vol. 184, Jul. 2020.

[34] S. J. Kazempour, and A. J. Conejo, "Strategic Generation Investment Under Uncertainty Via Benders Decomposition", IEEE Transactions on Power Systems, vol. 27, no. 1, pp. 424-432, Feb. 2012.

[35] J. R. Birge, and F. V. Louveaux, "A multicut algorithm for two-stage stochastic linear programs", European Journal of Operational Research, vol. 34, no. 3, pp. 384-392, Mar. 1988.

[36] Reliability System Task Force, ”The IEEE reliability test system-1996: A report prepared by the reliability test system task force of the application of probability methods subcommittee", IEEE Transactions on Power Systems, vol. 14, no. 3, pp. 1010-1020, Aug. 1999.

[37] [Online]. Available: https://github.com/FlexGrid/DER_Investment_ Planning

[38] "IEEE 33-bus test distribution system", 2000. [Online]. Available http: //ewh.ieee.org/soc/pes/dsacom/testfeeders/index.html

[39] I. Pena, C. Brancucci, and B. M. Hodge, "An Extended IEEE 118-Bus Test System High Renewable Penetration", IEEE Transactions on Power Systems, vol. 33, no. 1, pp. 281-289, Jan. 2018.

[40] J. Fortuny-Amat, B. McCarl, "A representation and economic interpretation of a two-level programming problem", Journal of Operational Research Society, vol. 32, no. 9, pp. 783-792, Sep. 1981. 